\title{
EXTENDED PAPER
}

Funktionale Analyse mittlerer Reichweite als Methode neuer kulturgeschichtlicher Kommunikationsforschung: Methodologisch-erkenntnistheoretische Begründung und Anwendungsbeispiele

Functional Analysis as Method of New Cultural Historical Communication Research. Methodology, Epistemology and Sample Applications

Corinna Lüthje 
Dr. Corinna Lüthje, Kommunikationswissenschaftlerin, Hamburg, Kontakt: corinna.luethje(at)gmail.com 


\title{
EXTENDED PAPER
}

\section{Funktionale Analyse mittlerer Reichweite als Methode neuer kulturgeschichtlicher Kommunikationsforschung: Methodologisch-erkenntnistheoretische Begründung und Anwendungsbeispiele}

\author{
Functional Analysis as Method of New Cultural Historical \\ Communication Research. Methodology, Epistemology and \\ Sample Applications
}

\section{Corinna Lüthje}

Zusammenfassung: Die Verbindung von Kommunikationsgeschichte und neuer Kulturgeschichte hat sich bisher im deutschen Sprachraum nicht etabliert. Die Gründe sind vor allem in methodischen und erkenntnistheoretischen Problemen verankert. Das Dilemma bezieht sich auf die Integration von komplexen Strukturen und individuellen Akteuren in Wandlungsprozessen. In diesem Aufsatz wird mit der funktionalen Analyse eine eigentlich altbekannte, aber selten empirisch angewendete Methode als Lösung vorgeschlagen. Nach der Klärung des Funktionsbegriffs wird die Theorie komplexer dynamischer Systeme (nach Klaus Mainzer) vorgestellt, die sich durch einen geringen Grad an Elaboration auszeichnet. Für jede zu untersuchende Form von System muss eine eigene Begrifflichkeit gefunden werden. Für die Anwendung auf soziokulturelle Phänomene folgt daraus, dass eine Anpassung an eine gesellschafts- und kulturtheoretische Epistemologie Vorbedingung für die Adaption ist. In diesem Aufsatz wird dafür der Strukturkonstruktivismus von Pierre Bourdieu vorgeschlagen, gefolgt von den methodologischen Grundlagen der funktionalen Methode (Robert K. Merton und Niklas Luhmann). Schließlich werden mit Medienproduktgeschichte und Rekonstruktion der Bildung des sozialen Gedächtnisses zwei Anwendungsbeispiele gebracht. Medienproduktgeschichte ist ein Beispiel für Medienwandel und für ein originär kommunikationswissenschaftliches, aber intradisziplinär zu realisierendes Forschungsprogramm. Das soziale Gedächtnis hingegen wird in Zusammenhang mit Hazardkommunikation thematisiert. Hier geht es um die Funktionen medialer Kommunikation im gesellschaftlichen Wandel. Das Konzept eines regionalen Geohazard ist nicht nur interdisziplinär, sondern durch die Integration von Natur-, Sozial- und Kulturwissenschaften inhärent fächergruppenübergreifend, wobei Kommunikation das Schlüsselprinzip darstellt. Die funktionale Analyse ist in diesem Fall auch eine Handreichung für das Gelingen interdisziplinärer Zusammenarbeit. Den Schluss des Aufsatzes bildet eine kritische Bewertung der Leistungsfähigkeit und Praktikabilität der Methode.

Schlagwörter: Funktionale Methode, Theorie komplexer dynamischer Systeme, Strukturkonstruktivismus, Medienwandel, soziale Erinnerung, Hazardkommunikation 
Abstract: The combination of the history of communication and the new cultural history has not been established in communication research so far. The reasons are rooted primarily in methodological and epistemological problems. The dilemma refers to the integration of complex structures and individual actors in the processes of change. The functional analysis is a well-known method. However, it is rarely used in empirical research. In this paper, the functional analysis (in the interpretation of Robert K. Merton and in the early work of Niklas Luhmann) is proposed as a suitable method for the solution of the problem mentioned above. The epistemological framework consists of two parts: the complex dynamical systems theory (in the interpretation of Klaus Mainzer) and Pierre Bourdieu's structural constructivism. The complex dynamical systems theory is characterized by a low degree of elaboration. For any type of system, a specific terminology has to be defined. Adaptation of a social and cultural theoretical epistemology is a precondition for the application of the complex dynamical systems theory on socio-cultural phenomena. An appropriate epistemology is Bourdieu's structural constructivism. Finally, two application examples are given. Firstly, the history of media products is an example of media change. This is an intra-disciplinary research program within communication science. Secondly, the communicative construction of social memory is addressed in the context of hazard communication. The concept of a regional geohazard is not only interdisciplinary, but inherently crossing subject-groups through the integration of natural sciences, social sciences and cultural studies. However, communication is the key principle. In addition, the method of functional analysis is assistance for successful interdisciplinary collaboration. The conclusion of the paper provides a critical assessment of the potential and practicability of the method.

Keywords: functional method, complex dynamical system, structural contructivism, media change, social memory, hazard communication

\section{Einleitung}

$\mathrm{Zu}$ Medienkulturgeschichte gibt es eine umfangreiche medienwissenschaftliche Literatur. Medienkulturgeschichte wird einerseits mit Bezug auf Einzelmedien betrieben, hier sollen als Beispiele die Kulturgeschichte des Radios von Hans Jürgen Koch und Hermann Glaser (2005) und die Medienkulturgeschichte des christlichen Predigers von Carsten Winter (2006) dienen. Andererseits wird die medienübergreifende Entwicklung thematisiert, wie z. B. in der bisher fünfbändigen Geschichte der Medien als Medienkulturgeschichte von Werner Faulstich (1996, 1997, 1998, 2002, 2004). Obwohl, wie S. J. Schmidt (vgl. 2003, S. 145) anmerkte, Mediengeschichte immer auch Kommunikationsgeschichte ist, hat sich Kommunikationsgeschichte als Kulturgeschichte bzw. Kulturgeschichte als Kommunikationsgeschichte bisher im deutschen Sprachraum nicht etabliert So bilanzierte Rainer Gries, dass die „Dreierbeziehung ,Kultur“, ,Kommunikation“ und ,Geschichte“ gänzlich missraten zu sein [scheint]“ (2008, S. 46). Dies gilt insbesondere für die neue Kulturgeschichte. Ein wenig konsterniert stellte er fest, dass sowohl in der Bestandsaufnahme der „New Cultural History“ von Peter Burke (2005) als auch in dem „Kompendium Kulturgeschichte“ von Ute Daniel (2001) weder Kommunikationsgeschichte noch Kommunikation thematisiert werden (vgl. Gries, 2008, 
S. 45). Gries“ Aufsatz „Kulturgeschichte des Kommunizierens“ erschien 2008 in dem von Klaus Arnold et al. herausgegebenen, als „diskursives Hand- und Lehrbuch“ bezeichneten Sammelband „Kulturgeschichte - Positionen und Werkzeuge“. Diese Anthologie ist als Bestandsaufnahme der deutschsprachigen kommunikationshistorischen Forschung nach der Jahrtausendwende zu lesen. Und - obwohl sich einige wenige Autorinnen und Autoren in Bereiche der neuen Kulturgeschichte begeben, wie Susanne Kinnebrock zu Gender, Markus Behmer zu Oral History oder Christoph Classen zu qualitativen Diskursanalysen - tatsächlich dominieren sozialhistorische Zugänge das Bild. Auch die im gleichen Jahr in der zweiten, ergänzten Auflage erschienenen „Grundzüge der Medien- und Kommunikationsgeschichte" von Jürgen Wilke (2008) sind streng der sozialhistorischen Tradition verpflichtet. Die verschüttete kulturhistorische Fundierung der Kommunikationsgeschichte (und vice versa) ist nicht in der Ignoranz der neuen Kulturgeschichte zu suchen, sondern ist einerseits in der Fachgeschichte der deutschen Kommunikationswissenschaft und andererseits in methodischen sowie erkenntnistheoretischen Problemen verankert.

Maria Löblich (2010) datiert die empirisch-sozialwissenschaftliche Wende des Fachs Kommunikationswissenschaft auf die 1960er Jahren. Letztlich handelt es sich aber um einen langwierigen Prozess, um einen Kampf um die symbolische Macht, dessen Nachwirkungen heute noch in den aktuellen Selbstverständnisund Methodendiskussionen zu spüren sind. Die geisteswissenschaftlichen Wurzeln des Fachs wurden zunehmend negiert. Mit dem Label einer empirischen Sozialwissenschaft war nicht nur die Abwendung von der Hermeneutik und die Zuwendung zur Empirie verbunden, sondern auch die absolute Bevorzugung quantitativer Methoden und Vernachlässigung qualitativer empirischer Methoden. Als Gegenbewegung entwickelte sich seit den 1970er Jahren die geistes- und literaturwissenschaftlich fundierte Medienwissenschaft zu einer eigenständigen Disziplin. Die Abgrenzung zur „kulturwissenschaftlichen“ Medienwissenschaft mit dem Zweck der Stärkung der eigenen disziplinären Identität beförderte das sozialwissenschaftliche Selbstverständnis der Kommunikationswissenschaft. Fachgeschichte ist jedoch nicht nur evolutionstheoretisch mit Umweltbedingungen bzw. Anforderungen der Umwelt verbunden, sondern auch in hohem Maße mit individuellen Akteuren an Schlüsselpositionen. Löblich selbst weist darauf hin, dass „fünf der sechs Institute (...) Mitte der 1960er Jahre von Professoren mit empirisch-sozialwissenschaftlicher Orientierung geleitet (...) wurden. Ihre ersten Entscheidungen betrafen in der Regel die Bibliothek und die Lehrinhalte." (2010, S. 553) Es waren also Individuen, die zwar nach bestimmten Kriterien selektiert wurden, die dann aber eine eigenständige Entscheidungsmacht hatten und damit sowohl das gegenwärtig legitime Wissen als auch mit den Lehrinhalten die Gestaltung der Zukunft bestimmten. Die erstaunliche Homogenität der Entscheidungen ist auf übereinstimmende habituelle Dispositionen zurückzuführen. Die Integration dieser Akteure in die strukturelle Betrachtung steht in der Kulturgeschichte der Kommunikationswissenschaft noch aus.

Ein ähnlicher Prozess war in den deutschen Geschichtswissenschaften zu beobachten. Als „Gegenposition gegen die dominante Sozial- und Gesellschaftsgeschichte (...), die sozioökonomische Strukturen und Prozesse, Organisationen und 
Institutionen als das Wesentliche der Geschichte betrachtete" (Daniel, 2003, S. 187), formierte sich seit den 1960er Jahren im internationalen Raum eine neue Richtung der Kulturgeschichte. Im angelsächsischen Sprachraum wurde diese neue Bewegung erstmals 1989 programmatisch als New Cultural History durch die Anthologie von Lynn Hunt gefasst (vgl. Peltonen, 2004, S. 205) und nach der Jahrtausendwende von Peter Burke (2005) aktualisiert. Auch in den deutschen Sprachraum diffundierte die neue Forschungsrichtung, jedoch zunächst unter verschiedenen Bezeichnungen wie Historische Anthropologie oder Alltags- und Mentalitätsgeschichte und erst mit Verspätung, seit Beginn der 1990er Jahre, mit der „kulturalistischen Wende“ unter dem Label „Kulturgeschichte“. Ute Daniel (vgl. 2003, S. 198) macht für diesen deutschen Sonderweg die Dominanz der „Bielefelder Schule“ der Sozial- und Gesellschaftsgeschichte verantwortlich, die den „Kern der Sozialgeschichte“ definierte als „Strukturen und Prozesse, die hinter dem Rücken der Menschen den Gang der Geschichte im wesentlichen ausmachten und denen gegenüber die sog. ,weichen Faktoren', also Wahrnehmungsweisen und Symbolwelten, als sekundär zu gelten hätten“ (ebd.). In der deutschsprachigen Kommunikationswissenschaft kam die kulturalistische Wende mit noch größerer Verspätung an, hier über den Umweg der Cultural Studies, die seit Ende der 1990er Jahren zunehmend Akzeptanz fanden, bei gleichzeitiger Negierung der deutschen kulturwissenschaftlichen Tradition. Trotzdem kann seit der Jahrtausendwende von einer allmählichen Rückbesinnung auf die kulturwissenschaftlichen Wurzeln gesprochen werden. Ein Beispiel dafür ist die Anthologie „Kulturwissenschaft als Kommunikationswissenschaft", herausgegeben von Matthias Karmasin und Carsten Winter (2003).

Die neue Kulturgeschichte zeichnet sich nicht durch ein einheitliches Paradigma, sondern durch ein plurales Theorien- und Methodenverständnis aus (vgl. Daniel, 2003, S. 198; Nünning, 2005, S. 165; Raphael, 2003, S. 229). Die große Klammer ist das primäre Interesse für „symbolische Formen der Vergangenheit [...]: Zeichen, Metaphern, politische Sprachen, kollektive Repräsentationen oder Rituale“ (Raphael, 2003, S. 228) bzw. für „kulturelle Sinnbildungen und die Ordnungen des Wissens" (Jaeger, 2004, S. 536). Stark beeinflusst wurde die neue Kulturgeschichte von den Cultural Studies, Praxistheorien (Pierre Bourdieu), postmoderne Theorien (Michel Foucault) und der Ethnologie bzw. Kulturanthropologie (Clifford Geertz). Aber auch ältere Konzepte der Kulturgeschichte (Norbert Elias: Kultur als Prozess) wurden neu gelesen und integriert (vgl. Raphael, 2003, S. 233235). Mit dem linguistic Turn wurde ein semiotischer Kulturbegriff begründet (Hunt, 1998). Wirklichkeit wird als sprachlich (also kommunikativ) konstituiert angesehen (vgl. Nünning, 2005, S. 166). Neben biographischen Interviews (Oral History) und Diskursanalyse ist die dichte Beschreibung eine wichtige Methode. Quantifizierende Methoden werden kaum angewendet (vgl. ebd., S. 165). Im Zentrum stand zunächst die Perspektive einzelner Akteure und Oral History sowie Macht und von der früheren Geschichtswissenschaft marginalisierte Gruppen (vgl. ebd., S. 167). Mit Oral History war auch ein starker Fokus auf Zeitgeschichte verbunden (vgl. Jordan, 2010, S. 200 sowie Bösch \& Danyel, 2012). Ein wichtiges Beispiel dafür ist Frauen- und Geschlechtergeschichte (vgl. Raphael, 2003, S. 237). Auch das Verhältnis von Geschichte und Gedächtnis wird zunehmend 
thematisiert (vgl. Jordan, 2010, S. 172). Gesellschaftliche Eliten waren mit Bezug zur Machtfrage von Beginn an im Fokus der neuen Kulturgeschichte. Seit Ende der 1990er Jahre finden auch wieder Aspekte der Politik (vgl. ebd., S. 241-242) und der „sozialen Verankerung kulturgeschichtlicher Phänomene“ (Nünning, 2005, S. 167) Beachtung. Nicht nur die individuelle Mikro-, sondern auch die strukturelle Makroebene werden betrachtet. Neu ist zudem der spatial Turn, mit dem die Verknüpfung von geographischem Raum und soziokultureller Entwicklung möglich wird (vgl. Jaeger, 2004, S. 538). Bisher fehlen jedoch in der auf qualitative Methoden konzentrierten neuen Kulturgeschichte adäquate Methoden zur Integration der unterschiedlichen Ebenen (vgl. z. B. Confino, 1997).

In diesem Aufsatz wird eine eigentlich altbekannte und in der deutschsprachigen Kommunikationswissenschaft stark diskutierte, aber selten empirisch angewendete Methode als Lösung vorgestellt. Die funktionale Methode ist im Prinzip schon in der historischen Methode von Karl Lamprecht (1912) angelegt gewesen, wobei Lamprecht nach dem Stand der Zeit zwar bereits von systemischen Zusammenhängen, aber gleichzeitig noch von der linearen und nahezu zwingenden Entwicklung eines historischen „Normalverlaufs“ (vgl. ebd., S. 73-138) ausging. Vom Anfang bis zur Mitte des 20. Jahrhunderts war die funktionale Analyse eine gebräuchliche Methode in praktisch ,allen Wissenschaften vom Menschen: Biologie und Psychologie, Wirtschafts- und Rechtswissenschaften, Anthropologie und Soziologie." (Merton, 1967, S. 136) In der deutschsprachigen Kommunikationswissenschaft ist die funktionale Methode eng mit einer soziologischen Systemtheorie verbunden, wahlweise mit dem normativen Strukturfunktionalismus von Talcott Parsons oder mit dem Äquivalenzfunktionalismus von Niklas Luhmann. Beide Großtheorien haben den Anspruch, die soziale Welt als Ganzes zu erklären. Parsons' Strukturfunktionalismus wurde vor allem von Ulrich Saxer in der Kommunikationswissenschaft vertreten und auch für sein Konzept von „MedienKulturkommunikation“ verwendet (1998). Im Zentrum seines Interesses standen Medien als Organisation (vgl. Winter, 2003, S. 74). Allerdings lockerte er in seinem Spätwerk „Mediengesellschaft“ den „Strukturdeterminismus“ zu Gunsten des „dynamischeren Äquivalenzfunktionalismus von N. Luhmanns funktionalstruktureller Systemtheorie“ (Saxer, 2012, S. 53). Mit der funktionalen Methode können kultur-, kommunikations- und medienhistorische Zusammenhänge untersucht werden, wie Faulstich in seiner Medienkulturgeschichte zeigt. Im Mittelpunkt seiner Analyse steht nicht die Struktur der Medienorganisation, sondern die ästhetische Struktur, allerdings nicht die Ästhetik eines einzelnen Mediums, sondern die Gesamtkonstellation der Medien (vgl. Winter, 2003, S. 77). Medienkultur ist für ihn ein „kontingent-historischer funktionaler Zusammenhang von Mediensystemen“ (Winter, 2012, S. 196). Auch Faulstich wandte sich im Laufe seiner wissenschaftlichen Arbeit Luhmanns Systemtheorie zu. Während er zu Beginn der 1990er Jahre noch die These vertrat, „Medien sind nicht Vermittlungsinstrumente, sondern Gestaltungsinstrumente (...). Kultur heiße demnach: kreativer Umgang mit den Medien zum Zweck der Konstruktion von Wirklichkeit“ (Faulstich, 1991, S. 10), spielen Handelnde „in Faulstichs späterer funktionaler Systemtheorie keine große Rolle mehr. Sie werden vielmehr durch spezifische Annah- 
men über die Selbststeuerung und Selbstorganisation von Medien-Systemen überflüssig gemacht." (Winter, 2003, S. 77)

Aufgrund der breiten Rezeption von Luhmanns Systemtheorie in der deutschsprachigen Kommunikationswissenschaft sollte man annehmen, dass auch die funktionale Methode weit verbreitet wäre. Dies ist jedoch ein Trugschluss, sie wird selten in der empirischen Forschung eingesetzt. Manfred Rühl merkte an, dass „in der jüngeren Vergangenheit wenig Grundlagenforschung (...) in der Kommunikationswissenschaft [passiert]. Deshalb wahrscheinlich der oft saloppe Umgang mit Grundlagenbegriffen. Auf diese Weise verbaut sich die Kommunikationskommunität leicht interdisziplinäre Kontakte ebenso wie eine eigenständige Diskussion der Probleme spezifischer Theoriedesigns. “ (2008, S. 143) Ein Beispiel für den saloppen Umgang mit Grundbegriffen ist der Funktionsbegriff, der häufig selbst von systemtheoretisch verorteten KommunikationswissenschaftlerInnen nicht in der soziologischen Bedeutung als Folge, sondern wie in der Umgangssprache als Aufgabe, Ziel, Zweck oder Rolle benutzt wird. Dies liegt an der Konvergenz von sprachlichen Symbolen und einer nachlassenden Rezeption von Primärtexten. Für Rühl (vgl. ebd., S. 135-143 und 2011, S. 155) ist die funktionale Methode (in Anlehnung an Luhmann) eine „Theorietechnik“ und damit eine Chance für die Kommunikationswissenschaft zur eigenständigen Theoriebildung und auch der intradisziplinären Integration. Gründe für die seltene Anwendung der funktionalen Methode in kommunikationswissenschaftlicher Forschung können der methodeninhärente hohe Komplexitätsgrad, die (trotz aktueller Methodendiskussion) immer noch vorhandene Priorisierung von quantitativen Erhebungsmethoden in der Kommunikationswissenschaft, die damit verbundene eher positivistische Grundhaltung und daraus folgend die marginalisierte Fähigkeit der Theorieformulierung im Fach sein. Ein anderes Problem ergibt sich aus der scheinbaren Kopplung der funktionalen Methode an soziologische Großtheorien. Die beiden Leitautoren Parsons und Luhmann haben nicht empirisch gearbeitet. ,Theorietechnik' scheint daher mit Verzicht auf Empirie verbunden zu sein. Abgesehen von diesen Gründen liegen kaum Anleitungen zur funktionalen Methode vor. Auch Rühl belässt es bei erkenntnis- und methodentheoretischen Überlegungen. Faulstichs Medienkulturgeschichte ist eine Ausnahme, die jedoch zeigt, dass die funktionale Analyse das geeignete Instrument ist, das „methodische Problem der wechselseitigen Kausalität von Medienentwicklung und sozialem Wandel“ (Münch \& Schmidt, 2005, S. 202) zu lösen, ohne dabei dem „technologiedeterministischen Fehlschluss“ zu verfallen, bei dem „soziale Wirkungen ausschließlich aus technischen Merkmalen eines Mediums geschlossen oder zumindest diesen maßgeblich zugeschrieben" werden (ebd., S. 203). Allerdings wird Faulstichs medienwissenschaftliche Arbeit in der Kommunikationswissenschaft weitgehend ignoriert.

Faulstichs Medienkulturgeschichte ist eher in der traditionellen Kulturgeschichtsforschung denn in der neuen Kulturgeschichte verankert. Aus der Perspektive der neuen Kulturgeschichte ist Luhmanns strukturelles Denken und Ignoranz der Akteursperspektive problematisch für die Anwendung der funktionalen Methode. Carsten Winter (2003) hat versucht, dieses Problem durch die Verbindung von Giddens Strukturationstheorie und Halls Artikulationstheorie zu lösen. Letztlich bleibt dieser Versuch auf der Ebene des theoretischen Diskurses und gibt 
keine Hinweise zur empirischen Umsetzung. Außerdem verknüpft er die Theoriediskussion über den Zusammenhang von Medienentwicklung und Gesellschaftswandel und den Anteil von individuellen Akteuren daran mit Globalisierung und damit mit einem Großthema, anstatt zunächst die grundsätzlichen Parameter seines Entwurfs zu verdeutlichen. Zudem fehlt eine Definition des zentralen Funktionsbegriffs. Richtig und wichtig ist jedoch, dass die funktionale Methode nicht exklusiv für das Theorem des (späten) Luhmanns nach der autopoietischen Wende reserviert, sondern auch auf andere Theorien über systemischen Zusammenhänge und ihre Dynamik anwendbar ist.

In diesem Text soll nicht die lückenlose Rekonstruktion des kommunikationswissenschaftlichen Theoriediskurses der letzten fünf Dekaden über den Funktionalismus geleistet werden. Ebenso sollen in diesem Aufsatz nicht die Begriffe ,Kommunikation', ,Medien' und ,Kultur' geklärt werden. Die vorherigen Ausführungen sollen lediglich dazu dienen, den Problembereich grob abzustecken. Dieser Aufsatz ist methodologisch ausgerichtet. Er hat das Ziel, die funktionale Analyse als geeignete Methode für die Integration von neuer Kulturgeschichte und Kommunikationsgeschichte in der empirischen Forschung und zur Theoriegenerierung darzustellen, die Operationalisierung zu erläutern und das Leistungsspektrum der Methode aufzuzeigen. Die bisherigen Überlegungen zusammenfassend lässt sich jedoch sagen, dass es dafür einige epistemologische Probleme zu lösen gilt. Dies sind:

(a) das Problem der theoretischen Integration von Akteursperspektive und systemischer Struktur,

(b) das Problem der Theorie-Empirie-Integration,

(c) das Problem des Strukturdeterminismus sowie

(d) die Dimensionierung der Fragestellung.

Zur Lösung dieser Probleme wird in diesem Aufsatz die epistemologisch-methodologische Verknüpfung von drei Bausteinen vorgeschlagen:

(1) Das Forschungsprogramm für funktionale Analysen mittlerer Reichweite nach Robert K. Merton mit methodologischen Ergänzungen aus dem Frühwerk von Niklas Luhmann,

(2) die Theorie komplexer dynamischer Systeme (oder kurz: Komplexitätstheorie), hier in der Lesart von Klaus Mainzer und

(3) der Strukturkonstruktivismus als erkenntnistheoretische Quintessenz von Pierre Bourdieus reflexiver Gesellschaftstheorie.

\section{Einordnung der theoretischen Bausteine und weiterer Ablauf}

Mertons Beitrag zur Entwicklung der funktionalen Analyse wird in der kommunikationswissenschaftlichen Methodendiskussion häufig unterschätzt. So nennt Manfred Rühl in seiner sonst sehr sorgfältigen Rekonstruktion des Funktionalismus Merton zwar korrekt als Urheber der Unterscheidung zwischen manifesten und latenten Funktionen sowie zwischen Eufunktionen und Dysfunktionen (vgl. 2008, S. 138). Als Quelle für den Äquivalenzfunktionalismus nennt er jedoch einzig Luhmann (vgl. ebd., S. 139). Fakt ist jedoch, dass der Begriff der funktionalen Alternativen bzw. Äquivalenzen auf Merton zurückgeht. Merton wandte sich 
gegen den Strukturfunktionalismus der Sozialanthropologie von Radcliffe-Brown und Malinowsky und der Soziologie von Parsons. Ebenso wandte er sich vom Ideal der allumfassenden Großtheorie ab und war stattdessen bestrebt, Theorien mittlerer Reichweite zu entwickeln. Trotzdem war er sich bewusst, dass es sich bei seinem Konzept nicht um eine ,Neuerfindung', sondern um eine Weiterentwicklung auf der Basis der ,Klassiker' handelte. Das große Verdienst Mertons war, den Begriff der ,Funktion' im soziologischen Gebrauch klar von der Bedeutung in anderen Disziplinen abzugrenzen. Doch auch in der Soziologie wurde und wird der Begriff unterschiedlich genutzt. Deshalb ist es wichtig, in jedem Beitrag zur funktionalen Analyse die Verwendung dieses Kernbegriffes klar zu definieren. Merton löste den Begriff der Funktion als Folge von der intendierten Zielgerichtetheit in der Bedeutung als Rolle. Weiter erkannte er, dass Funktionen für Erhalt eines größeren Zusammenhangs von Elementen (also eines Systems) von verschiedenen Elementen wahrgenommen werden können und ein Element verschiedene Funktionen haben kann. Damit führte er den Begriff der funktionalen Äquivalenz ein. Weiter erkannte er, dass es neben positiven Funktionen auch Dysfunktionen gibt, die in der Analyse berücksichtigt werden müssen, und trennte manifeste von latenten Funktionen. Nach Mertons Vorstellung sollten soziologische Theoriebildung und empirische Forschung eng verbunden sein. Merton integrierte theoretische und empirische Wissenschaft nicht in seiner Person. Der „problemorientierte Theoretiker" arbeitete jedoch an der Columbia Universität in New York eng mit dem "problemorientierten Empiriker" Paul F. Lazarsfeld zusammen (vgl. Coser, 2000, S. 156). Die Zusammenarbeit im „Office of Radio Research“, das 1944 in „Bureau of Applied Social Research“ umbenannt wurde, dauerte von 1941 bis zu Lazarsfelds Tod 1976. Nach Mertons eigener Schätzung sprachen er und Lazarsfeld durchschnittlich 10 bis 15 Stunden in der Woche miteinander (vgl. Simonson, 2005, S. 12). 1944 hielten beide zusammen ein Graduiertenseminar ab, in dem sie eine Agenda zur Verknüpfung von Theorie und empirischer Forschung entwickelten (vgl. ebd., S. 12-13). Merton und Lazarsfeld betrieben zusammen bis in die 1950er Jahre Kommunikationsforschung und veröffentlichten als Koautoren mehrere Aufsätze, von denen „Mass Communication, Popular Taste, and Organized Social Interaction“ (1948) besonders herausragt. Mit Bezug auf die damalige Medienlandschaft arbeiteten die beiden Autoren drei soziale Funktionen von Massenmedien aus (status conferral function, enforcement of social norms und narcotizing dysfunction). Merton wird von Klaus Merten (2007, S. 333) zusammen mit Lazarsfeld im „Stammbaum der Wirkungsforschung“ aufgeführt. Doch davon abgesehen wird sein Werk in der gegenwärtigen Kommunikationswissenschaft wenig rezipiert. In diesem Aufsatz soll jedoch nicht Mertons Kommunikationsforschung gewürdigt werden, sondern sein Beitrag zur funktionalen Methodologie. Wichtig ist aber, zu berücksichtigen, dass diese Methodologie zwar nicht mit kommunikationswissenschaftlichen Termini arbeitet, aber dass sie im engen Kontext mit Kommunikationsforschung entstand (vgl. Simonson, 2005). Merton machte, wie Lewis A. Coser (vgl. 2000, S. 158) feststellte, die funktionale Analyse mittlerer Reichweite zu einem flexiblen Instrument der Theoriegenerierung auf empirischer Basis. Das Vorgehen bei der funktionalen Analyse mittlerer Reichweite erinnert dabei einerseits an die Grounded Theory der Chicagoer Schule. Ande- 
rerseits wird bei der funktionalen Analyse eine Vielzahl von Daten unterschiedlicher Herkunft berücksichtigt. Diese dichte Datensammlung erinnert an das Vorgehen der Dichten Beschreibung von Clifford Gertz. Zudem berücksichtigt Merton die wechselseitigen Auswirkungen von individuellem Handeln und sozialen Zwängen. Doch auch Mertons Programm hat aus heutiger Sicht Schwächen. Einmal liegt dies an seinem kantianischen Bild vom Menschen als animal rationale, der sich jederzeit seiner Handlungen bewusst ist, aber in diesen Handlungen von bestehenden Strukturen bestimmt wird. Handeln ist nach Merton also strukturell determiniert, der Handelnde hat aber die bewusste Wahl, diesen Strukturen zu entsprechen oder ihnen zuwider zu handeln und danach die Konsequenzen zu tragen. Dieses Problem soll später mit der Integration von Bourdieus Strukturkonstruktivismus gelöst werden. Eine weitere Schwäche liegt in der Fixierung auf die Funktionen eines bestimmten Elements auf den Gesamtzusammenhang. Zur Lösung dieses Problems wird auf das Frühwerk von Niklas Luhmann zurückgegriffen.

In der „Kölner Zeitschrift für Soziologie und Sozialpsychologie“ erschien 1962 Luhmanns erster Aufsatz über Funktion und Kausalität. Luhmann hielt sich zwischen 1960 und 1961 (also noch vor Beginn seiner wissenschaftlichen Karriere) in Harvard auf und lernte dort Parsons kennen. Der Aufsatz entstand unter dem Eindruck dieses Aufenthalts. Luhmann bezog sich darin auch auf Merton, z. B. bei der Nennung des Begriffs der funktionalen Äquivalenz (vgl. 2005, S. 17). Der ,frühe' Luhmann nahm den Gedanken des Äquivalenzfunktionalimus wieder auf und entwickelte ihn weiter. Luhmann wies darauf hin, dass Funktionen nicht nur äquivalent sein können, sondern die einzelnen Elemente im Gesamtzusammenhang miteinander in Verbindung stehen. Auf jedes Element wirken verschiedene andere Elemente ein. Die Wirkung ist nicht monokausal auf ein einzelnes Element zurückzuführen, sondern holistisch auf viele verschiedene Einflüsse. Luhmann interessieren die Wirkungen des Gesamtzusammenhangs auf ein bestimmtes Element oder auf den Systemerhalt. Nicht der Äquivalenzfunktionalismus, sondern die Einführung von Wechselwirkungen und damit die Relativierung des Kausalitätsprinzips ist die entscheidende Ergänzung von Luhmann. Die Methode der funktionalen Analyse des ,frühen Luhmanns' (vor der autopoietischen Wende) ist durch ihre Objektzentrierung und Prozessorientierung, auch beim Weg der Erkenntnis selbst, hervorragend geeignet zur Zusammenführung der Ergebnisse von verschiedenen Teilstudien zu den einzelnen Elementen. Luhmanns spätere Systemtheorie wird jedoch in diesem Aufsatz aufgrund der bereits vorher benannten Kritik an der Ignoranz individueller Akteure aber auch wegen seiner Vorstellung von geschlossenen Systemen nicht verwendet. Wir verlassen an dieser Stelle die ,Evolutionsgeschichte' der soziologischen Systemtheorie und machen einen Zeitsprung von ein paar Jahrzehnten.

S. J. Schmidt hat 1991 festgestellt, dass mit der Vorstellung von Systemen ein großes Potenzial zur Konzeptualisierung komplexer Zusammenhängen in der Forschung verbunden ist. Er kam zu dem Schluss, dass „auch der Einsatz systemtheoretischer Modelle (...) erkennen [läßt], daß diese terminologisch ,hochgerüstet' werden müssen, um schon mit der ,auf der Hand liegenden' Komplexität von „Kultur’ fertigzuwerden“ (Schmidt, 1991, S. 31). An dieser Stelle wird dieser Ein- 
schätzung widersprochen. Gerade angesichts überbordender Komplexität erscheint (ganz im Sinne von Merton) der Versuch sinnlos, eine allumfassende Großtheorie soziokultureller Phänomene aufstellen zu wollen. In diesem Aufsatz wird ,terminologisch abgerüstet` und als Gegenmodell für das Grundverständnis von systemischen Zusammenhängen die Theorie komplexer dynamischer Systeme vorgeschlagen, die anschlussfähig ist zum dynamisch-transaktionalen Ansatz von Werner Früh und Klaus Schönbach (1982). Die Theorie komplexer dynamischer Systeme gilt als neueste Variante der Systemtheorien. Sie findet als Nachfolgerin der Chaostheorie vor allem in den Umweltwissenschaften zunehmend Verbreitung (Lewin, 1993). Sie ist „eine interdisziplinäre Methodologie zur Modellierung nichtlinearer Prozesse in Natur und Gesellschaft" (Mainzer, 2004, S. 32, vgl. auch Bar-Yam, 1997, S. 1). Komplexitätsforschung versteht sich als integrative Wissenschaft: „Komplexitätsforschung führt unterschiedliche Denkansätze zusammen, die aus den verschiedenen Wissenschaften gewonnen werden. (...) Komplexitätsforschung beschäftigt sich fachübergreifend mit der Frage, wie durch Wechselwirkungen vieler Elemente eines komplexen Systems (z. B. Moleküle in Materialien, Zellen in Organismen oder Menschen in Märkten und Organisationen) Ordnungen und Strukturen entstehen können, aber auch Chaos, Spannungen und Konflikte in komplexen Systemen zu erkennen und ihre Ursachen zu verstehen, um daraus Einsichten für neue Gestaltungspotentiale der Systeme zu gewinnen.“ (Mainzer, 2008, S. 10) Ihren Ursprung hat die Komplexitätsforschung im Santa Fe Institut (New Mexico), das 1984 gegründet wurde. Die Mehrzahl der Gründungsmitglieder waren Physiker vom Los Alamos National Laboratory. Im Gegensatz zu den soziologischen Systemtheorien (und damit gewöhnungsbedürftig für Sozialwissenschaftler) kann die Komplexitätstheorie nicht auf einen Leitautor zurückgeführt werden, sondern ist eher als eine kollektive Entwicklung zu betrachten. Diesem Aufsatz liegen vor allem Schriften des Wissenschaftstheoretikers Klaus Mainzer zugrunde. Für Mainzer ist menschliche Gesellschaft das komplexeste System, „das wir derzeit überhaupt kennen“ (ebd., S. 12) aufgrund der Verhaltenskomplexität der individuellen Menschen, die als relativ eigenständige Systemelemente betrachtet werden. Die Theorie komplexer dynamischer Systeme zeichnet sich durch einen extrem geringen Grad an Elaboration aus. Sie ist reduziert auf die Benennung von prinzipiellen Elementen und Prozessen. Dies macht sie, ebenso wie die funktionale Analyse, zu einem flexiblen, auf vielfältige Phänomene in nahezu allen Disziplinen und interdisziplinären Zusammenhängen anwendbaren Instrument. Gleichzeitig ist es mit diesem puren, auf das Wesentliche reduzierten Konzept möglich, hochkomplexe Zusammenhänge zu erklären. Der begrifflichen Geschlossenheit und Starre der soziologischen Großtheorien setzt sie Offenheit und Anpassungsfähigkeit entgegen. Für jede zu untersuchende Form von System muss jedoch eine eigene Begrifflichkeit gefunden werden bzw. muss die Definition der Begriffe angepasst werden. Für die Anwendung auf soziokulturelle Phänomene bedeutet dies, dass eine Anpassung an eine gesellschafts- und kulturtheoretische Epistemologie Vorbedingung für die Adaption der Komplexitätstheorie ist. In diesem Aufsatz wird dafür der Strukturkonstruktivismus von Pierre Bourdieu vorgeschlagen, in dem Akteure und soziale Struktur als interdependent angesehen werden. Damit werden die Probleme des Strukturdetermi- 
nismus und der Integration von Individuum und Struktur gelöst. Zudem hat Bourdieu auf die Ausformulierung einer Großtheorie verzichtet, aber die interdependenten Einzelelemente seiner Vorstellung von Kultur und Gesellschaft als flexible Instrumente aufgefasst sowie strikte Theorie-Empirie gefordert und betrieben.

Dieser methodologische Text ist im Folgenden zweigeteilt in (a) die Darstellung der drei epistemologischen und methodologischen Theorieelemente, die für die Anwendung der funktionalen Methode im Sinne einer neuen kulturbistorischen Kommunikationsforschung relevant sind und darauf folgend (b) die Darstellung von zwei Anwendungsbeispielen. Diese Anwendungsbeispiele sollen als lockere Leitfäden zur Durchführung von funktionalen Analysen dienen. Mit ihrer Hilfe wird der (mögliche) Ablauf von Studien dargestellt. Es handelt sich aber nicht um wörtlich zu lesende Anleitungen mit strikten Vorgaben, weil jedes neue Projekt seine eigenen Anforderungen hat, auf die mit der Wahl der Erhebungsmethoden und der Begrifflichkeit eingegangen werden muss. Weil das Ziel dieses Aufsatzes nicht die Entwicklung einer allgemeinen Theorie von Medien, Kommunikation und Kultur ist und weil die drei Theorieelemente jeweils als flexible Instrumente verstanden werden müssen, die auf eine Vielzahl von Problemstellungen angewendet werden können, werden im Theorieteil vor allem Primärtexte exzerpiert. Damit soll eine zwar verkürzte, aber trotzdem möglichst unverfälschte Darstellung erreicht werden. Die Integration der Elemente erfolgt in der Anwendung. Mit den beiden Anwendungsbeispielen Medienproduktgeschichte und Rekonstruktion der Bildung des sozialen Gedächtnisses aus der Forschungspraxis der Autorin soll das Leistungsspektrum der funktionalen Analyse für neue kulturhistorische Kommunikationsforschung angedeutet werden. Medienproduktgeschichte, in diesem Fall die Programmgeschichte von Klassik Radio, ist ein Beispiel für Medienwandel und damit für ein originär kommunikationswissenschaftliches, aber intradisziplinär zu realisierendes Forschungsprogramm. Im zweiten Beispiel wird die Funktion von medialer Kommunikation bei der Bildung des sozialen Gedächtnisses in Zusammenhang mit Hazardforschung thematisiert. Das Konzept eines regionalen Geohazard ist nicht nur interdisziplinär, sondern durch die Integration von Natur-, Sozial- und Kulturwissenschaften inhärent fächergruppenübergreifend, wobei Kommunikation das Schlüsselprinzip darstellt. Hier geht es um den Beitrag medialer Kommunikation zum gesellschaftlichen Wandel. Die funktionale Analyse ist in diesem Fall auch eine Handreichung für das Gelingen interdisziplinärer Zusammenarbeit. Den Schluss des Aufsatzes bildet eine kritische Bewertung der Leistungsfähigkeit und Praktikabilität der Methode.

\section{Funktionsbegriff: Folge (Merton und Luhmann)}

Wichtig waren für Merton klar definierte Begriffe und ein „analytisches Paradigma, das der Erhebung und Verarbeitung des Materials eine systematische und methodische Kontrolle auferlegt" (Hartmann, 1967, S. 118). Der Schlüsselbegriff für eine funktionale Analyse ist der ihr zugrundeliegende Funktionsbegriff. Merton (1967) hat fünf wesentliche Bedeutungen von Funktion in verschiedenen Kontexten klassifiziert: 
1. Öffentliche Versammlung: An erster Stelle steht der im englischen Sprachraum populäre Gebrauch „im Sinne öffentlicher Versammlungen oder festlicher Anlässe, und zwar meist solchen mit zeremoniellem Beiwerk" (ebd., S. 120). Diese Bedeutung des Begriffs ist im deutschen Sprachraum irrelevant.

2. Beruf: Im Bereich der Wirtschaftswissenschaften wird der Begriff „faktisch zum Synonym von ,Beruf'“ (ebd.). Funktionale Analyse bedeutet hier die Herausarbeitung der Verteilung von Berufen in einer Gruppe. Merton schlägt vor, diese Analyse treffender als „Berufsanalyse“ (ebd.) zu bezeichnen, um Verwirrung zu vermeiden.

3. Tätigkeit: Drittens wird in der Politikwissenschaft Funktion mit „dem Inhaber einer sozialen Position, insbesondere dem Inhaber eines Amtes oder einer politischen Stellung, zugewiesenen Tätigkeiten “ (ebd.) gleichgesetzt.

4. Variable: In der Mathematik bezeichnet Funktion „eine Variable (...), die in Beziehung zu einer oder mehreren anderen Veränderlichkeiten steht, durch welche letztere die erstere Variable ausgedrückt werden kann, oder von deren Wert ihr eigener Wert abhängt“" (ebd., S. 121).

5. Prozess: In der Biologie wird Funktion als Bezeichnung für die „vitalen oder organischen Prozesse, insofern sie zur Erhaltung des Organismus beitragen“ (ebd.), verstanden.

Die Basis der soziologischen funktionalen Analyse ist für Merton mit der Zusammenführung der mathematischen und der biologischen Bedeutung des Wortes gelegt. Er bezieht sich kritisch auf die funktionalistische Anthropologie Alfred Radcliffe-Browns. Für diesen waren Einzelmenschen die wesentlichen Einheiten der Sozialsphäre und durch Netze sozialer Beziehungen zu einem integrierten Ganzen verbunden. Funktion war für ihn jedoch die Rolle jeder wiederkehrenden Tätigkeit im sozialen Leben als Ganzem und ihr Beitrag zur Erhaltung der strukturellen Kontinuität (vgl. Merton, 1967, S. 122). Auch für Bronislaw Malinowski waren Funktionen Rollen (von anthropologischen Tatsachen), die „sie innerhalb des Gesamtsystems der Kultur spielen, mittels der Art und Weise, in der sie innerhalb des Systems aufeinander bezogen sind“ (ebd.). Mit dem Begriff der Rolle als Funktion ist für Merton jedoch die Gefahr verbunden, dass er synonym mit Ausdrücken wie „Zweck, Motiv, Intention, Ziel“ (ebd., S. 123) verwendet wird und ,Interdependenz' (aus dem mathematischen Funktionsbegriff) sowie ,Prozess' (aus dem biologischen Funktionsbegriff) nicht mehr genügend Berücksichtigung finden (vgl. ebd., S. 122). Diese beiden Begriffe sind später entscheidend für die Anwendung des Funktionsbegriffs auf komplexe dynamische Systeme.

Für Merton ist der Begriff Funktion notwendig vom Standpunkt der Beobachter und nicht vom Teilnehmer definiert. „Soziale Funktion bezieht sich auf beobachtbare objektive Konsequenzen, nicht auf subjektive Dispositionen (Ziele, Motive, Zwecke).“ (1967, S. 124) Damit wird es möglich, „unvorhergesehene Folgen sozialen Zweckhandelns“ (Hartmann, 1967, S. 119) zu analysieren. Merton geht weiter davon aus, dass „fortdauernde kulturelle Formen einen Saldo funktionaler Folgen aufweisen, entweder für die Gesellschaft als eine Einheit oder für Untergruppen mit genügend Macht, um diese Formen durch direkten Zwang oder indirekte Manipulation intakt zu halten“ (1967, S. 131), dass es sich also um einen 
historischen Prozess des Wachsens und Wandels handelt. Dabei sind, laut Merton, Funktionen nicht universell. Sie hängen von den jeweiligen strukturellen Voraussetzungen $\mathrm{ab}$ und sind wandelbar. Dies unterscheidet, wie später Luhmann anmerkt, ein soziales System klar von einem biologischen Organismus: „Aus einem Esel kann keine Schlange werden (...) Eine Sozialordnung kann dagegen tiefgreifende strukturelle Änderungen erfahren, ohne ihre Identität und ihren kontinuierlichen Bestand aufzugeben.“ (2005, S. 23)

Dabei ist es wichtig, nicht nur positive Funktionen zu analysieren, sondern auch negative Funktionen, also Dysfunktionen, denn was für eine Gruppe funktional ist, kann für andere innerhalb der gleichen Gesellschaft dysfunktional sein (vgl. Merton, 1967, S. 127). „Funktionen sind diejenigen beobachtbaren Folgen, die die Anpassung eines gegebenen Systems fördern; Dysfunktionen sind diejenigen beobachtbaren Folgen, die die Anpassung des Systems mindern. Außerdem sind nichtfunktionale Folgen möglich, die für das in Betracht gezogene System einfach irrelevant sind.“ (Ebd., S. 140) Für den Bestand eines sozialen Systems kann das gleiche Objekt mehr als eine Funktion haben und die gleiche Funktion kann durch unterschiedliche Objekte erfüllt werden. Damit wird der Begriff der funktionalen Äquivalente bzw. funktionalen Alternativen oder funktionalen Substitute eingeführt (vgl. ebd., S. 133).

Für Luhmann ist in diesem Sinne der Funktionsbegriff eine Folge und „ein regulatives Prinzip für die Feststellung von Äquivalenzen im Rahmen funktionaler Variablen“ und ersetzt damit den „kausalwissenschaftlichen Funktionalismus durch einen Äquivalenzfunktionalismus“ (Luhmann, 2005, S. 19). Monokausalität ist damit ausgeschlossen: „Die funktionalistische Aussage betrifft nicht die Beziehung von Ursache und Wirkung, sondern ein Verhältnis mehrerer Ursachen zueinander bzw. mehrerer Wirkungen zueinander, also die Feststellung funktionaler Äquivalenzen.“ (Ebd., S. 22) Damit sind die für die Anwendung des Funktionsbegriffs auf komplexe dynamische Systeme wichtigen Wechselwirkungen integriert.

Für die funktionale Analyse ist es erforderlich, „eindeutig zwischen subjektiven Kategorien der Disposition und objektiven Kategorien der beobachtbaren Folgen zu unterscheiden“ (Merton, 1967, S. 125). Das schließt aber Ziele, Zwecke und Motive nicht aus. Merton unterscheidet weiter zwischen latenten und manifesten Funktionen. Manifeste Funktionen sind „diejenigen objektiven Folgen, die zur Anpassung des Systems beitragen und von Beteiligten im System sowohl beabsichtigt als auch wahrgenommen werden “ (ebd., S. 141), sie sind also mit Zielen, Zwecken und Motiven übereinstimmend. Diese Funktionen sind intendiert. Um sie empirisch zu belegen, genügt es, Aussagen der Beteiligten über ihre Absichten mit Folgen zu vergleichen. Latente Funktionen hingegen sind dementsprechend solche, „die weder beabsichtigt sind noch wahrgenommen werden " (ebd., S. 141), sie sind also unintendiert und invisibel. Manifeste Funktionen können in latente Funktionen umgewandelt werden, also invisibel werden (vgl. Bourdieus Habitus- und Praxistheorie).

Funktion wird zusammenfassend hier definiert als manifeste oder latente Folge von Einzelelementen in Interaktion mit anderen Einzelelementen auf einen größeren Zusammenhang von Elementen oder auf andere Einzelelemente in diesem Zusammenhang, die von Zielen, Zwecken und Motiven zunächst unabhängig ist, aber unter bestimmten Umständen mit ibnen identisch sein kann. 


\section{Systembegriff: Theorie komplexer dynamischer Systeme (Klaus Mainzer)}

Das Erkenntnisziel der funktionalen Analyse ist nicht die Substanz der Einzelelemente: „Oft ist es nicht wichtig, was ein Erkenntnisgegenstand nun genau ist, sondern (...) was er bewirkt. Dies gilt insbesondere für solche Prozesse, die nicht nur durch ihre Elemente, sondern vielmehr durch die Relation zwischen diesen Elementen charakterisiert werden können: für Systeme." (Merten 2007, S. 33) Mit dem Funktionsbegriff wird die Dynamik von Prozessen greifbar gemacht. Es ist jedoch auch wichtig, als zweiten Schlüsselbegriff den Systembegriff und damit das Verständnis der Struktur zu klären.

Ein komplexes dynamisches System besteht aus einer Vielzahl von Elementen (bzw. Agenten), die auf eine bestimmte Art und Weise angeordnet sind und in dieser Anordnung miteinander in einem Zusammenhang stehen. Dieser Zusammenhang von Elementen bildet ein einfaches System und ist damit die Vorstufe des komplexen dynamischen Systems bzw. die erste Stufe der Beobachtung. „Die mikroskopischen Zustände der Elemente bestimmen den makroskopischen Zustand des Systems.“ (Mainzer, 2004, S. 4) Die einzelnen Elemente fügen sich selbstständig und selbstorganisiert zu "wohlgeordneten und funktionierenden Einheiten“ zusammen (vgl. ebd., S. 6). Das System als Ganzes und in seinen Elementen ist veränderlich. Die zweite Stufe ist die Beobachtung dieser Veränderungen über die Zeit. Das System wird als dynamisch wahrgenommen. „Die Dynamik des Systems, d. h. die Änderung der Systemzustände in der Zeit, wird durch Differentialgleichungen beschrieben, wobei jeder zukünftige Zustand durch den Gegenwartszustand eindeutig bestimmt ist." (Ebd., S. 4) Diese Dynamik wird durch Interkationen der Elemente hervorgerufen. Bei ähnlichen Ursachen und Wirkungen handelt es sich um dynamische lineare Systeme. „Mathematisch erhalten wir dann eine lineare Gleichung. Eine Lösung dieser Bewegungsgleichung lässt sich als Zeitreihe des Orts in Abhängigkeit von der Zeit darstellen. (...) Eine Kausalitätsanalyse ist in diesem Fall nicht nur vollständig durchführbar, sondern auch berechenbar." (Ebd., S. 2) Wichtig ist für die Berechnung der linearen Dynamik lediglich die strikte Kontrolle der Ausgangsbedingungen (vgl. ebd., S. 3). Komplexe dynamische Systeme zeichnen sich jedoch durch Nichtlinearität ihrer Veränderungen aus. Komplexe Systeme bestehen aus gekoppelten (,interconnected“) oder verflochtenen (,interwoven“) Teilen (vgl. Bar-Yam, 1997, S. 1), die nicht nur miteinander interagieren, sondern wechselwirken. System und Einzelelemente sind interdependent. „Die gleichzeitige Wechselwirkung vieler Elemente wird durch nichtlineare Funktionen erfasst. Zufallsereignisse (...) werden durch zusätzliche Fluktuationssysteme berücksichtigt.“ (Mainzer, 2004, S. 5) Weiterhin zeichnen sich komplexe dynamische Systeme durch Emergenz aus, womit „neue Verhaltensweisen“ des Systems gemeint sind, die „den einzelnen Bestandteilen nicht [direkt] zugeordnet werden können“ (Brandau, 2010, S. 1). Emergenz ermöglicht die Selbstorganisation von Ordnung. „Dabei kommt es zu charakteristischen Rückkopplungen von Systemelementen, bei denen Wirkungen von Ursachen selber wieder zu Ursachen werden. So entstehen makroskopische Strukturen, die nicht durch die Systemelemente vorgegeben sind, aber durch ihre Wechselwirkung bei geeigneten Anfangs- und Nebenbedingungen (d. h. Einstellung von Kontrollpara- 
metern) möglich werden. (...) Die Zirkelkausalität dieser vielfältigen Wechselwirkungen wird mathematisch durch gekoppelte nichtlineare Gleichungen der einzelnen Systemelemente ausgedrückt." (Mainzer, 2004, S. 4) In dynamischen komplexen Systemen interagieren nicht nur die integrierten Elemente. Es handelt sich grundsätzlich um offene Systeme, die „im Stoff- und Energieaustausch mit ihrer Umwelt sind“ (ebd., S. 8). Sie sind adaptive und lernende Systeme, die sich ihren Umweltbedingungen anpassen.

Wie Mainzer (vgl. 2004, S. 2) anmerkt, erlauben nichtlineare Gleichungen nicht immer eine genaue Berechenbarkeit. Er zeigt die „Grenzen der praktischen Berechenbarkeit von Wirkungen aus Ursachen bei nichtlinearer Dynamik" auf (ebd., S. 3), die zu Unsicherheit bzw. gewusstem Nichtwissen führen. Dies liegt einerseits an der schwierigen Kontrolle der Anfangsbedingungen, andererseits an dem steigenden Komplexitätsgrad der verfügbaren Daten - was sowohl die Anzahl von interagierenden Elementen als auch die breite Ausdifferenziertheit der Daten betrifft. Das zurzeit wohl populärste Beispiel dafür ist die Klimaforschung und auch Ulrich Becks Konzeption der „Weltrisikogesellschaft“ (2007) stimmt mit dieser Argumentation überein. Mainzer (2004) selbst stellt fest: „Im Zeitalter der Globalisierung erweisen sich Länder und Kulturen als komplexe Systeme, die den Gesetzen nichtlinearer Dynamik folgen.“ (S. 34) Mit Blick auf die Kulturgeschichte verweist Mainzer (vgl. ebd., S. 18) darauf, dass die Theorie von dynamischen komplexen Systemen geeignet ist, die Entwicklung menschlicher Gesellschaften zu verstehen. Mikroebene (individuelle Entscheidungen einzelner Menschen) und Makroebene (kollektive Prozesse und Strukturen) sind theoretisch integriert (vgl. ebd., S. 19). Der einzelne Mensch wird als Systemelement aufgefasst. Bereits 1997 hat Yaneer Bar-Yam Gesellschaft als Beispiel für komplexe Systeme aufgeführt, mit individuellen Menschen und Technologie als relevanten Elementen sowie Kommunikation als primäre Form der Interaktion: 
Abbildung 1: Beispiele für komplexe Systeme

\begin{tabular}{|c|c|c|c|c|}
\hline System & Element & Interaction & Formation & Activity \\
\hline Proteins & Amino Acids & Bonds & Protein folding & $\begin{array}{l}\text { Enzymatic } \\
\text { activity }\end{array}$ \\
\hline $\begin{array}{l}\text { Nervous system } \\
\text { Neural networks }\end{array}$ & Neurons & Synapses & Learning & $\begin{array}{l}\text { Behavior } \\
\text { Thought }\end{array}$ \\
\hline Physiology & Cells & $\begin{array}{l}\text { Chemical } \\
\text { messengers } \\
\text { Physical support }\end{array}$ & $\begin{array}{l}\text { Developmental } \\
\text { biology }\end{array}$ & $\begin{array}{l}\text { Movement } \\
\text { Physiological } \\
\text { functions }\end{array}$ \\
\hline Life & Organisms & $\begin{array}{l}\text { Reproduction } \\
\text { Competition } \\
\text { Predation } \\
\text { Communication }\end{array}$ & Evolution & $\begin{array}{l}\text { Survival } \\
\text { Reproduction } \\
\text { Consumption } \\
\text { Excretion }\end{array}$ \\
\hline $\begin{array}{l}\text { Human } \\
\text { economies } \\
\text { and societies }\end{array}$ & $\begin{array}{l}\text { Human Beings } \\
\text { Technology }\end{array}$ & $\begin{array}{l}\text { Communication } \\
\text { Confrontation } \\
\text { Cooperation }\end{array}$ & Social evolution & $\begin{array}{l}\text { Same as Life? } \\
\text { Exploration? }\end{array}$ \\
\hline
\end{tabular}

Quelle: Bar-Yam, 1997, S. 8.

Bar Yam (vgl. ebd., S. 4) nennt die Familie als Beispiel für ein dynamisches komplexes soziales System: Es handelt sich um eine Zusammenstellung von Individuen, wobei jedes Individuum in Beziehung zu den anderen Individuen steht. Es gibt ein Wechselspiel zwischen den Beziehungen und den Eigenschaften der jeweiligen Individuen. Die Familie muss mit der Außenwelt interagieren; außerdem gibt es unterschiedliche Formen von Familie (Kernfamilie, Kleinfamilie, Großfamilie etc.). Der Zustand sowohl der gesamten Familie (System) als auch ihrer einzelnen Mitglieder (Systemelemente) ist veränderlich in Abhängigkeit der Entwicklung der Beziehungen zueinander und zur Außenwelt. Diese Veränderungen sind nicht vorhersagbar, die Dynamik ist nichtlinear. Die Besonderheit von dynamischen komplexen sozialen Systemen liegt darin, dass „wir in der Regel über keine Bewegungsgleichungen für das individuelle Verhalten der Systemelemente auf der Mikroebene" verfügen (Mainzer, 2004, S.19). Auch Brandau (vgl. 2010, S. 1-2) stellt fest, dass, neben der Komplexität durch eine Vielfalt von Einflussgrößen, subjektive Wahrnehmungsdifferenzen relevant für individuelle Entscheidungen sind. Im Modellierungskonzept der Soziodynamik wird aus diesem Grund normalerweise methodisch die Mikroebene ausgeklammert, sich auf die Makroebene konzentriert und zur Berechnung der quantitativen Modelle auf statistische Daten zurückgegriffen. Letztlich ist dieses Vorgehen aber dem integrativen Potential der Theorie dynamischer komplexer sozialer Systeme nicht angemessen. Mainzer (2004, S. 20) überlegt, auf der Mikroebene Gleichungen individueller Gehirndynamiken zugrunde zu legen, kommt aber zu dem Schluss, dass dieser Ansatz wegen der Komplexität solcher Gleichungen nicht praktikabel wäre. Außerdem 
sieht er die Interdependenz von individuellen Entscheidungen und (kollektiver) Struktur.

In diesem Sinne können komplexe dynamische soziokulturelle Systeme als Figurationen aufgefasst werden. Figurationen sind „Gruppen oder Gesellschaften verschiedener Art", die von interdependenten Menschen gebildet werden (Elias, 1997, S. 90) und damit „Interdependenzgeflechte“ (ebd., S.71). In Nobert Elias' Begriff der Figuration geht es um eine doppelte Interdependenz, einmal der Abhängigkeit der beteiligten Individuen voneinander und andererseits der gegenseitigen Abhängigkeit von Individuum und Gesellschaft, die sich gegenseitig die Bedingung der Möglichkeit sind. Elias' Vorstellung von Kultur als Prozess betont die inhärente Dynamik einer Figuration: das Interdependenzgeflecht einer Figuration unterliegt Wandlungen. Damit wird der Bogen von der Kultursoziologie zur Kulturgeschichte geschlagen und die Theorie der komplexen dynamischen Systeme kann zum Verständnis dieser Prozesse beitragen: „Geschichte lässt sich in Phasenübergängen verstehen, die an Instabilitätspunkten in neue Ordnungen umschlagen, die wiederum instabil werden können, um neuen Ordnungen Platz zu machen. Nichtlineare Dynamik bedeutet aber, dass wir diese Prozesse nicht in allen Details zentral steuern können. (...) Ihre Gesetze verstehen bedeutet nicht, sie berechnen und beherrschen zu können. Sensibilität für empfindliche Gleichgewichte ist eine neue Qualität der Erkenntnis nichtlinearer Dynamik. “ (Mainzer, 2004, S. 34)

Die Konsequenz aus den bisherigen Überlegungen ist, dass die Analyse von komplexen dynamischen soziokulturellen Systemen nicht quantitativ auf Wahrscheinlichkeitsberechnungen, sondern qualitativ auf das Verständnis der Prozesse der Wechselwirkungen bzw. Funktionen ausgerichtet sein muss. Außerdem geht es nicht nur um Netzwerke oder relationale Verknüpfungen, sondern auch (oder vor allem) um symbolische Formen und kollektive Symbole.

Für die empirische Untersuchung von dynamischen komplexen Systemen sollten folgende zentrale Merkmale berücksichtigt und in Zusammenhang gebracht werden (vgl. Bar-Yam, 1997, S. 5):

- Elemente (und ihre Anzahl)

- Interaktionen (und ihre Stärke)

- Anordnung der Elemente und Funktionen

- Vielfalt und Veränderlichkeit

- Umwelt (und ihre Anforderungen)

- Vorgänge und ihre Folgen

Für die qualitative Modellierung bietet sich die funktionale Methode an. Zuvor müssen jedoch erkenntnistheoretische Grundlagen geklärt werden. Die Integration von Akteurs- und Strukturperspektive fällt im deutschen Sprachraum infolge der Nachwirkungen des Positivismusstreits zwischen Kritischem Rationalismus und Konstruktivismus nicht leicht. Zudem muss (wenn jedes Individuum als Systemelement betrachtet wird) auch der oder die Forschende sich selbst als Systemelement in die Modellierung integrieren. Eine Lösung bietet die strukturkonstruktivistische Kultur- und Gesellschaftstheorie von Pierre Bourdieu. 


\section{Strukturkonstruktivismus (Bourdieu)}

„Hätte ich meine Arbeit in zwei Worten zu charakterisieren, das heißt, wie es heute oft geschieht, sie zu etikettieren, würde ich von strukturalistischem Konstruktivismus oder von konstruktivistischem Strukturalismus sprechen.“ (Bourdieu, 1992b, S. 135) Schon im Begriff des Strukturkonstruktivismus ist die Interdependenz von Akteur (Mikro) und Struktur (Makro) wörtlich angelegt und wird durch die vice versa-Formulierung von Bourdieu noch betont. Menschen werden in eine soziokulturelle Struktur hineingeboren, erfahren dadurch eine primäre Prägung was ihre Wahrnehmungs-, Handlungs- und Wirkungsmuster betrifft, wandern im Laufe ihres Lebens durch diverse Strukturen und bauen diese in ihre individuellen Muster ein. Gleichzeitig verändern diese Individuen die Strukturen, mit denen sie in Berührung kommen. Diese interdependenten Prozesse sind normalerweise invisibilisiert, d. h. sie entziehen sich der Wahrnehmung der beteiligten Akteure. Mit drei Sätzen lässt sich die Interdependenz von sozialem Akteur und sozialer Struktur beschreiben, die den Kern von Pierre Bourdieus soziokulturellem Theorieuniversum ausmacht. Tatsächlich handelt es um komplizierte und komplexe Prozesse, die Bourdieu in seinen „partiellen Theorien des Sozialen“ (Rehbein, 2006. S. 9) (Habitus, Praxis, Kapital, Klassen, symbolische Macht, sozialer Akteur, Feld und Raum) sichtbar macht, wobei diese einzelnen Theoriekomponenten in einem inneren Zusammenhang stehen. Dieser innere Zusammenhang könnte durchaus selbst als komplexes dynamisches System begriffen werden. Neben dieser gesellschaftstheoretischen Konzeption wendet er den Strukturkonstruktivismus konsequent auch wissenschaftsphilosophisch und erkenntnistheoretisch an (vgl. Bourdieu, 1992a, S. 8-10.). Weitere Gründe für die Angemessenheit der Anwendung des Strukturkonstruktivismus auf die Modellierung von komplexen dynamischen Systemen sind:

- Die verschiedenen Teilkonzepte (Elemente) sind interdependent und organisieren sich selbst zu einem komplexen dynamischen System (Gesamtkonzept, Theorie).

- Durch selbstreflexiver Einstellung und strikte Theorie-Empirie-Integration wird der Wissenschaftler selbst in das qualitativ zu modellierende System integriert.

- Durch die Variabilität der Begriffe und große Bandbreite von soziokulturellen Phänomenen ist Vielfalt gegeben.

- Von Interesse sind nicht das vordergründig Sichtbare und von den Elementen direkt Steuerbare, sondern für Systemelemente selbst invisibilierte Prozesse; dies sind z. B. nichtintendierte Funktionen als Ergebnis von Nichtlinearität.

Loïc Wacquant (vgl. 2003, S. 107) hat auf Schwierigkeiten hingewiesen, die mit dem ,Erstkontakt' mit Bourdieus Denken verbunden sind. Zunächst ist mit Umfang und herausragender Vielfältigkeit seiner Schriften eine Hemmschwelle verbunden. Dieses Werk fordert sowohl vom Exegeten wie auch vom Leser, „das wissenschaftliche Begehren wie auch die wissenschaftlichen Kompetenzen zu erweitern“ (ebd., S. 108). Wacquant begründet dies mit Bourdieus bewusst transdisziplinärem Denken, das „nicht nur die kanonische Trennung zwischen Theorie 
und Empirie, sondern darüber hinaus auch die ,theologischen' Konflikte zwischen den theoretischen Gruppierungen ignoriert. (...) Es gibt beim Autor von Die feinen Unterschiede keinen Gegensatz zwischen der Ausarbeitung von Forschungskonzepten oder Erklären und Verstehen, der Analyse von Ursachen und der Interpretation der Gründe. Die Aporien des Positivismusstreits, die rechts des Rheins viel Tinte fließen ließen, lösen sich von selbst durch eine in der Praxis untrennbar verbundene theoretische und empirische Forschung." (Ebd., S. 108) Ein besonderes Merkmal von Bourdieus Forschungspraxis ist also die permanente TheorieEmpirie-Integration. Deren Besonderheit besteht darin, „dass sie von einer ständigen Reflexion über die Voraussetzungen des Möglichen und der Grenzen der soziologischen Erkenntnis angetrieben wird; diese wiederum sind dadurch bedingt, dass die soziologische Erkenntnis von einem besonderen Akteur produziert wird, dem Wissenschaftler, dem es darum geht, die Welt, von der er sich mit dem Ziel ihrer Beobachtung zurückgezogen hat, darzustellen: wie ein Schauspiel, das es zu entziffern gilt" (ebd., S. 108-109).

Substanzen und Objekte sind für Bourdieus Ansatz von untergeordneter Relevanz. Wichtiger sind für ihn Relationen und Beziehungen. Ernst Cassirer folgend wandte er sich gegen die „substanzialistische Denkweise, die dazu verleitet, keine andere Realität anzuerkennen als die der unmittelbaren Anschauung der Alltagserfahrung sich darbietenden: also Individuen und Gruppen“ (Bourdieu, 1992b, S. 138). Soziale Wirklichkeit (in Anlehnung an Durkheim) ist für ihn „ein Ensemble unsichtbarer Beziehungen in einem Raum mit wechselseitig sich äußerlicher Positionen, die sich wechselseitig zueinander definieren“ (ebd.). Dieses Prinzip bezog Bourdieu systematisch auf Individualität, gesellschaftliche Bereiche und Gesellschaft selbst (vgl. Papilloud, 2003, S. 28). Kurz gesagt ist seine Vorstellung von Gesellschaft zunächst die einer Sozialtopologie, eines sozialen Raums. Neben dem Raumbegriff verwendet Bourdieu auch den Begriff des sozialen Feldes. Für soziale Felder gelten die gleichen Regeln wie für den sozialen Raum (vgl. Papilloud, 2003, S. 35). Sie sind jedoch kleiner dimensioniert und bilden gesellschaftliche Teilbereiche ab (sozusagen ,Subräume'). Soziale Felder entwickeln sich, ihre Logiken und Funktionsweisen in relativer Autonomie. Sie sind eigenständig und klar abgegrenzt, stehen aber trotzdem in einem systemischen Zusammenhang und beeinflussen sich gegenseitig. Im sozialen Raum und darin in sozialen Feldern befinden sich Individuen (Akteure) und Gruppen von Akteuren. Sie positionieren sich innerhalb des Raums durch die Ansammlung von verschiedenen Kapitalsorten. In jedem dieser Felder ist eine bestimmte Mixtur von Kapitalien wichtig und diese verleiht darin „ihrem Träger Stärke bzw. Macht.“ (Bourdieu, 1985, S. 9) Kapital ist dabei nicht mit dem marxistischen Begriff identisch. Kapital ist grundsätzlich akkumulierte Arbeit, sowohl in materieller Form als auch in verinnerlichter, inkorporierter Form. Bourdieu unterscheidet vier Grundformen von Kapital: a) ökonomisches Kapital, b) kulturelles Kapital, c) soziales Kapital und d) symbolisches Kapital.

Ein soziales Feld ist immer auch ein Kraftfeld, d. h. ein „Ensemble objektiver Kräfteverhältnisse, die allen in das Feld Eintretenden sich als Zwang auferlegen“" (Bourdieu, 1985, S. 10). Es ist ein historisch konstituierter Spielraum mit spezifischen Institutionen und eigenen Funktionsgesetzen (vgl. Bourdieu, 1992c, S. 111). Die Felder sind von handelnden Menschen abhängig, die der ,illusio' und des 
Interesses bedürfen, um überhaupt motiviert zu sein, in ein bestimmtes Feld einzutreten und dessen Spiel mitzuspielen. Die verschiedenen Felder sind von einer je spezifischen Logik bestimmt, die zu einem feldspezifischen Habitus führt. Bourdieu entmaterialisiert den traditionellen Habitusbegriff. Habitus steht nun nicht mehr für bestimmte Erscheinungsformen, wie Kleidung, Sprache, Gewohnheiten, sondern für das Prinzip, das dahintersteht. Habitusformen sind „Systeme dauerhafter und übertragbarer Dispositionen“ (Bourdieu, 1993, S. 98). Sie sind gleichzeitig strukturierte und strukturierende Strukturen. Sie fungieren als „Erzeugungsund Ordnungsgrundlagen für Praktiken und Vorstellungen“ (ebd.) und sind „objektiv ,geregelt' und ,regelmäßig', ohne irgendwie das Ergebnis der Einhaltung von Regeln zu sein, und genau deshalb genau kollektiv aufeinander abgestimmt (...), ohne aus dem Handeln eines Dirigenten hervorgegangen zu sein.“ (ebd., S. 99). Der Habitus beschreibt die jeweiligen Wahrnehmungs-, Denk- und Handlungsschemata eines bestimmten Akteurs. Damit ist der Habitus auch ein ,System der Grenzen' innerhalb derer ein Akteur wahrnehmen, denken, aber auch praktisch handeln kann (vgl. Fröhlich, 1994, S. 38). Der Habitus ist akkumulierte Geschichte, denn „frühere Erfahrungen kondensieren sich in den Menschenkörpern als Wahrnehmungs-, Denk- und Handlungsschemata und bleiben so aktiv präsent" (ebd.) und gleichzeitig ein Abbild der im Lebenslauf absolvierten Positionen in den verschiedenen Feldern mit ihren jeweils spezifischen Logiken. Menschen wachsen in einem historisch gewachsenen und vorstrukturierten Symbolfeld auf. „Der Habitus (...) ist Produkt der gesamten individuellen Geschichte, aber auch, vermittels der bildenden frühkindlichen Erfahrungen, der gesamten kollektiven Geschichte der Familie und Klasse." (Bourdieu, 1992c, S. 116) Wichtig bei der Entstehung des Habitus ist der Prozess der Inkorporierung, in dem die prägenden Strukturen für den Akteur invisibilisiert werden. Habitus und Feld stehen in einer unmittelbaren Abhängigkeit oder ,Komplizenschaft', oder folgen einem ,Spiel-Sinn' (vgl. Bourdieu, 1992a, S. 115), der sich in einer einfachen Formel zusammenfassen lässt. Wenn die objektiven Chancen eines Feldes mit den habituellen Neigungen bzw. Dispositionen eines Akteurs deckungsgleich sind (weil sich diese durch die Einverleibung von Strukturen eines ähnlichen Universums gebildet haben), dann müssen sich Akteure nur ,ihrer Natur' (als das, was ihre Geschichte aus ihnen gemacht hat) überlassen, um zu tun, was an der jeweiligen Position in dem jeweiligen Feld getan werden muss. Dies bedeutet, dass umso weniger Anpassungsarbeit für den Akteur bei Feldeintritt zu tun ist, je näher sein ererbter und gewachsener (erarbeiteter) Habitus dem des Feldes ist. Dieser Akteur fühlt sich in dem neuen Feld ,wie ein Fisch im Wasser', wie in seiner natürlichen Umgebung und weiß instinktiv, wie er sich zu verhalten hat. Für einen ,Quereinsteiger' ist sehr viel mehr Anpassungsarbeit zu leisten. Hier ist weniger der Instinkt als vielmehr Beobachtung und Nachahmung gefragt, was mit einer größeren Bewusstheit verbunden ist. Im Laufe der Zeit wird das bewusste Beobachten und Nachahmen immer unbewusster, das Denken und Handeln wird ,natürlicher', weil der feldspezifische Habitus inkorporiert wird.

Dieser Habitus materialisiert sich in feldspezifischen Lebensstilen und Praktiken. Mit dem Konzept der Praxis integriert Bourdieu die Handlungsebene in sein Theoriegebäude. Handlungen unterliegen nicht der theoretischen, sondern der 
praktischen Vernunft. Bei vielen (nicht allen) menschlichen Handlungen gibt es keine bewussten Überlegungen und keine rationale Kalkulation, weil ihr Erzeugungsprinzip im Habitus zu suchen ist. Sie sind aber auch nicht rein mechanisch, sondern Produkt der dialektischen Beziehung zwischen einem sozialen Feld und dem Akteur in ihm. Hierarchisch nach dem Grad der Rationalisierung können bewusst-intellektualisierte, habitualisiert-praktische und instinktiv-reflexive Handlungen unterschieden werden. Die Motivation zu einer praktischen Handlung ist auf die Disposition zurückzuführen, deshalb ist eine Praxis keine Befolgung theoretisch konstruierter Regeln und strukturale Modelle der Erklärung reichen nicht aus. Ein wichtiges Merkmal von Praxis ist, dass sie nahezu unbewusst abläuft. Mit dem Praxiskonzept grenzt sich Bourdieu von Mertons Strukturdeterminismus ab. Die Logik des Feldes bestimmt Bourdieu nach einer Formel, die auch die gegenseitige Abhängigkeit der einzelnen Komponenten seiner Sozialtheorie zeigt (Bourdieu, 1987, S. 175):

\section{$[($ Habitus $)($ Kapital $)]+$ Feld $=$ Praxis}

Mit Habitus und Kapital bezeichnet Bourdieu die „wechselseitige Dynamik von Individualität und Gesellschaft" (Papilloud, 2003, S. 30), die das Feld in einem dialektischen Prozess wandeln und die über die materialisierte Form der Praxis rekonstruiert werden können.

Bourdieus Begriffe sind kontingent, weil sie eine doppelte Anpassung erfahren. Zum einen werden sie dem jeweiligen Erkenntnisstand des objektivierenden Subjekts (Beobachter) in seinem zeitlichen und räumlichen Kontext (Umwelt) angepasst. Zum anderen geht Bourdieu nicht von einer generellen Universalität von sozialen Tatsachen aus, sondern von einer nahezu ins Unendliche gehenden $\mathrm{Va}$ riationsbreite, die eine ständige Neubestimmung (oder Feinjustierung) der Begrif$f e$ (Elemente) mit Blick auf das jeweilige Forschungsobjekt erfordert und erlaubt. Auch ist es nicht für jede Fragestellung notwendig, alle Komponenten in die Untersuchung zu integrieren. Schwingel (2005, S. 19) spricht in diesem Zusammenhang von einem Ensemble theoretischer und methodischer ,Werkzeuge': „Die - epistemologisch wohl fundierte - Anwendung der Erkenntniswerkzeuge ist gewissermaßen ,perspektivisch', d. h. sie erfolgt entsprechend den jeweiligen forschungsleitenden Interessen. " Grundlage für dieses Vorgehen ist neben der selbstreflexiven Einstellung das Primat der Forschungsorientierung und der TheorieEmpirie-Integration. Theoretische Konzepte sind kein Selbstzweck, sondern primär Mittel zum Zweck der empirischen Untersuchung sozialer Wirklichkeit (vgl. ebd.). Ihr generatives Prinzip ist die „soziologische Wissenschaftstheorie als ein System von Prinzipien und Regeln, das alle wissenschaftlich fundierten Handlungen und Analysen, und nur diese, bestimmt“ (Bourdieu, 1974, S. 9). Der systematische Zusammenhang der einzelnen Komponenten (Einheit) wird nicht durch einen logisch geschlossenen kategorialen Rahmen (einer Gesellschaftstheorie), sondern auf der metatheoretischen Ebene hergestellt. Mit Hilfe dieser epistemologischen und logischen Prinzipien wird ein „System von Beziehungen und explikativen Schemata dieser Beziehungen“" konstruiert (vgl. ebd.). 
Nicht die „substanziellen Realitäten“, die normalerweise Gegenstand soziologischer Analysen sind, interessieren ihn. Diese substanziellen Realitäten sind Individuen und Gruppen, ihre praktisch erlebten Handlungen und die damit unmittelbar einhergehenden Repräsentationen wie Ideen, Erwartungen, Wissen, vorgestellte Ziele, Pläne usw. (vgl. Bourdieu, 1976, S. 148). Die phänomenologische Erkenntnisweise ihrer Oberfläche ist Gegenstand einer Spontansoziologie (Durkheim) oder eines Subjektivismus. In der wissenschaftlichen Beschreibung einer vorwissenschaftlichen Erfahrung trifft hier ein unreflektierter Beobachter auf ein Phänomen und begreift die für ihn sichtbare soziale Welt als einen natürlichen und selbstverständlich vorgegebenen Kosmos (vgl. ebd., S. 147). Schwingel (vgl. 2005, S. 45) weist darauf hin, dass Bourdieus Gebrauch von ,Phänomenologie' ganz wörtlich zu verstehen ist als Lehre von den Erscheinungen, die der alltäglichen oder wissenschaftlichen Erkenntnis direkt zugänglich sind: die Primärerfahrungen sozialer Akteure im Sinne einer „praktischen Auffassung der sozialen Welt" (Bourdieu, 1976, S. 148). Die Position des Erkenntnissubjektes ist innerhalb des Feldes angesiedelt. Erkenntnis beruht in diesem Fall auf unreflektierter, unmittelbarer Erfahrung und ist damit subjektivistisch.

Viel wichtiger ist für Bourdieu die Erforschung der Ebene hinter den von sozialen Akteuren und Wissenschaftlern direkt erfahrbaren Tatsachen, denn „vergessen wird auf diese Weise, daß die Wahrheit der Interaktion nie völlig in der Interaktion liegt, so wie sie sich dem Beobachter offenbart.“ (Bourdieu, 1992b, S. 139) Voraussetzung dafür ist ein doppelter Bruch. Bourdieu geht von der Strukturhomologie zwischen Forscher und Gegenstand der Erkenntnis bzw. dessen Konstruktion aus. Nur durch seine habituelle Disposition ist der Forscher in der Lage, ein bestimmtes Forschungsobjekt wahrzunehmen und eine bestimmte Frage zu stellen. Um der „Naivität ersten Grades“ (Bourdieu, 1998, S. 86) zu entgehen und die Bedingungen der Möglichkeit eines Phänomens zu rekonstruieren, muss der Forschende zunächst einmal seine eigene Position genau bestimmen und rekonstruieren, sich selbst also als objektivierendes Subjekt objektivieren und sich die Möglichkeiten und Grenzen seiner Erkenntnis bzw. seiner Einschreibung in das Objekt bewusst machen. Der nächste Schritt ist die Rekonstruktion eines ,Mehr' an Sinn, das den Akteuren nicht unmittelbar zugänglich ist und das über die Rekonstruktion des subjektiv gemeinten Sinns hinausgeht.

Das Gegenkonzept zum Subjektivismus ist die objektivistische Erkenntnisweise, die im klassischen Strukturalismus (in der Linguistik von Saussure, in der Anthropologie von Lévi-Strauss, im philosophischen Marxismus von Althusser oder auch in Parsons Strukturfunktionalismus) realisiert ist. Der Strukturalismus konstruiert Modelle objektiver, vom Willen und Bewusstsein der Akteure unabhängiger Relationen und „erstellt die - gewöhnlich ökonomischen oder linguistischen - objektiven Beziehungen, die die verschiedenen Praxisformen und deren Repräsentationen, d. h. im besonderen die praktische und stillschweigende primäre Erfahrung der vertrauten Welt, strukturieren - freilich um den Preis des Bruchs mit dieser primären Erfahrung, folglich mit den stillschweigend übernommenen Voraussetzungen, die der sozialen Welt ihren evidenten und natürlichen Charakter verleihen " (Bourdieu, 1976, S. 48). Der Forscher nimmt nun eine distanzierte und objektivierende Haltung gegenüber den Forschungsobjekten ein. Wenn dieser 
Bruch mit der Primärerfahrung jedoch (wie beim späten Luhmann) zu dem entgegengesetzten Extrem führt, die Akteure in den Strukturen zu vernachlässigen und die Strukturen als von den Akteuren unabhängig zu betrachten, so wandelt sich die soziologische Erkenntnis von objektiv zu objektivistisch. In der Antithese liegt die Gefahr der „Naivität zweiten Grades“ der „Halbschlauen“, die sich für „ernüchterte Ernüchterer“, als „desillusioniert und desillusionierend“ (Bourdieu, 1998, S. 86) halten, was für Bourdieu der Ursprung vieler wissenschaftlicher Irrtümer ist, weil die „kritisierte Illusion [des Subjektivismus] zur Realität gehört und also auch in das Modell Eingang finden muss, mit dem sie erklärt werden soll und das zunächst nur gegen sie konstruiert werden kann.“ (Ebd.) Der Objektivismus blendet genauso wie der Subjektivismus die Frage nach den Bedingungen der eigenen Möglichkeit der Erkenntnis aus und behauptet doch eine Überlegenheit in der Erkenntnisfähigkeit, erhebt Anspruch auf legitime Herrschaft (vgl. ebd.). Die Position des Erkenntnissubjektes in der objektivistischen Erkenntnisweise ist außerhalb des zu untersuchenden Feldes angesiedelt.

Die Synthese (,zweiter Bruch') von subjektivistischer (These) und objektivistischer (Antithese) Erkenntnisweise ist für Bourdieu die praxeologische Erkenntnisweise. Sie betrifft verschiedene Ebenen: zunächst die Forschungspraxis, die Positionierung der Forschenden, aber auch das Erkenntnisobjekt selbst. Die Position des Erkenntnissubjektes in der praxeologischen Erkenntnisweise ist innerhalb des Feldes. Aber durch konsequente Selbstreflexion und Bewusstmachung der eigenen ,Verstricktheit' in das Feld versucht sich das objektivierende Subjekt zu objektivieren, also für die Analyse virtuell nach draußen zu begeben.

Bourdieus Erkenntnistheorie ist eng mit seinem Blick auf die Gesellschaft verknüpft. Er sieht in den sozialen Akteuren mit ihrem Alltagswissen und ihren praktischen Erfahrungen die Konstrukteure der sozialen Welt (und der Wissenschaftler ist ein besonderer sozialer Akteur). Deshalb müssen sie gleichberechtigt neben den objektiven Strukturen in der soziologischen Analyse berücksichtigt werden. Das bedeutet, sowohl (gewachsene) Strukturen zu analysieren, als auch die Individuen, die in diesen Strukturen handeln und sie schaffen bzw. transformatorisch reproduzieren, zu berücksichtigen. Damit wird der Bogen geschlagen zur funktionalen Analyse von dynamischen komplexen soziokulturellen Systemen als Methode der neuen kulturhistorischen Kommunikationsforschung.

\section{Methode}

Das Ziel einer funktionalen Analyse ist die Feststellung funktionaler Äquivalenzen und Interdependenzen sowie die Aufdeckung latenter Funktionen, um die Frage beantworten zu können, wie ein bestimmtes Problem auch anders gelöst werden kann. Durch die Sichtbarmachung von Prozessen und Aufzeigung von Alternativen sollen neue Handlungsoptionen erschlossen werden. Das deckt sich mit dem Ziel der Modellierung von dynamischen komplexen Systemen, „frühzeitig die Ordnungsparameter (...) zu erkennen, die eine Dynamik dominieren können" (Mainzer, 2004, S. 22). Es deckt sich auch mit Bourdieus Ziel, das Inkorporierte und Invisible über Reflexion bewusst zu machen. Mertons funktionale Methode, Bourdieus Strukturfunktionalismus und die Komplexitätstheorie haben, 
neben der grundsätzlichen Flexibilität, die Gemeinsamkeit, der Aufklärung verpflichtet zu sein.

In der funktionalen Analyse werden die Folgen einzelner Elemente auf andere Elemente oder das Gesamtsystem untersucht. Die Folgen sind Produkte von Interaktionen, Kommunikationen oder Transaktionen der einzelnen Elemente untereinander, die aber nicht einfach zu einem Saldo addiert werden dürfen, sondern einer strengen, reflexiven, durch eine möglichst dichte Datensammlung gestützten und kontextualisierten Interpretation unterzogen werden müssen. Eine Funktion kann dabei nicht monokausal gedacht werden. Die einzelnen Elemente stehen in gegenseitigem Bezug von Wechselwirkungen oder ,chemischen' Reaktionen. Funktionen dürfen nicht akkumulativ, sondern müssen emulgativ gedacht werden und sind deshalb schwer zu rekonstruieren. Wichtige Referenz hierbei ist Niklas Luhmann: „Zwischen Ursachen und Wirkungen besteht in diesem Sinne eine Unbestimmtheitsrelation. (...) Für sie [die funktionalistische Kausaltheorie] sind exklusive Kausalgesetze allenfalls ein analytischer Grenzfall. (...) Die funktionale Analyse setzt ihren Grundbegriff nicht in Form einer empirischen Hypothese an. Das unterscheidet sie von jeder teleologischen oder mechanischen Erklärung. Es wird nicht vorausgesetzt oder angenommen, dass bestimmte Ursachen faktisch vorkommen und dadurch das Eintreten bestimmter Wirkungen erklären oder umgekehrt.“ (Luhmann, 2005, S. 22)

Funktionale Analyse ist laut Merton „Interpretation von Daten durch Herausarbeitung ihrer Folgen für größere Strukturen, in die sie eingebettet sind“ (1967, S. 136). Sie ist ein „geregeltes Interpretationsverfahren“ und hängt ab von der "Dreier-Allianz zwischen Theorie, Methode und empirischen Daten“ (ebd., S. 119; dies deckt sich mit Bourdieus Forderung nach Theorie-Empirie-Integration). Dabei beobachtet er das Problem der Determinierung bzw. der „ideologischen Voraussetzungen funktionaler Analysen“ (ebd., S. 144). „In welchem Aus$\mathrm{maß}$ führt die soziale Position des funktionalistischen Soziologen (z. B. gegenüber einem bestimmten ,Klienten', der eine Untersuchung in Auftrag gegeben hat) eher zu der einen oder anderen Formulierung eines Problems, wie weit beeinflußt sie seine Voraussetzungen und Begriffe, und wie weit begrenzt sie den Bereich der aus seinem Material abgeleiteten Folgerungen?" (Ebd.) Um dieser Gefahr zu entgehen, sind alle beteiligten Personen zur permanenten Selbstreflexion über die eigene habituelle Disposition und den Weg der Erkenntnis angehalten. Dies ist absolut mit Bourdieus Strukturkonstruktivismus und seiner Forderung der Objektivierung des objektivierenden Subjektes (praxeologische Erkenntnisweise) zu vereinbaren, aber noch nicht weitgehend genug, weil Merton (und das ist ein Widerspruch zu seinen Ausführungen zur Datensammlung) in seiner Anleitung zur funktionalen Analyse die Begriffsbildung an den Anfang der Analyse stellt und nicht (wie Luhmann) auf Hypothesen verzichtet und die Theorie aus der Analyse heraus entwickelt. Bourdieu hat den amerikanischen Strukturfunktionalismus zwar weitgehend ignoriert (und auch Luhmanns Systemtheorie nicht wahrgenommen), er hat sich aber in Der doppelte Bruch (1998) mit Mertons Wissenschaftssoziologie auseinandergesetzt. Er wirft ihm jedoch vor, mit seinem strong program in der Naivität des zweiten Grades, also dem Objektivismus zu verharren: „Merton wiederum trägt der Existenz des wissenschaftlichen Kosmos zwar 
Rechnung, wendet aber weiterhin wissenschaftliche Kategorien auf ihn an, die er sich aus eben dieser Welt vorschreiben lässt und gibt als Beschreibung der positiven Gesetze ihres Funktionierens aus, was nur eine Auflistung der normativen Regeln ist, zu denen man sich offiziell bekennt." (Bourdieu, 1998, S. 85) In der Forschungspraxis laufen jedoch Datensammlung und Theoriebildung parallel $\mathrm{ab}$ und beeinflussen einander. Das Schließen erfolgt in einer permanenten Abfolge von Induktion, Deduktion und Abduktion (vgl. Peirce, 1983). Zu den verwertbaren Daten können auch bereits bestehende Theorien und Forschungsergebnisse gehören, diese können sogar den Ausgangspunkt der funktionalen Analyse bilden. Obgleich es wichtig ist, die funktionale Methode nicht als hypothesentestendes, sondern als theoriegenerierendes Verfahren anzuwenden, werden im Laufe des Erkenntnisprozesse je nach Arbeitsstand Arbeitshypothesen formuliert, modifiziert oder verworfen.

Neben der reflexiven Einstellung soll eine möglichst dichte Datensammlung das Ergebnis absichern (dies zeigt die Nähe der funktionalen Methode zu Clifford Geertz' dichter Beschreibung, 1987). Merton betont, dass es der Vorzug der funktionalen Methode ist, „daß sie den Soziologen zur Sammlung von Angaben anregt, die sonst vernachlässigt werden könnten “ (1967, S. 144). Dabei ist auch diese Sammlung von Daten einem Prozess unterworfen. Innerhalb dieses Prozesses können plötzlich ganz neue Aspekte in den Blickwinkel kommen, die das anfängliche Forschungsdesign ad absurdum führen. Neben der Reflexivität auf die Erkenntnisgrundlagen der Forschenden ist Offenheit und Flexibilität im Bereich der Datenerhebung gefordert, um die Determinierung der Datensammlung zu vermeiden. „Beim Prozeß der Entwicklung einer funktionalen Interpretation wird der soziologische Analytiker sich stets noch nach anderen als den zu Beginn in Betracht gezogenen Daten umsehen müssen. Interpretation und Sammlung von Daten sind daher im Zusammenhang der Begriffe und der diese Begriffe verbindenden Sätze unauflöslich miteinander verbunden. “ (Merton, 1967, S. 144)

Jede funktionale Analyse wird mit einem festgelegten Bezugspunkt durchgeführt. Für Merton stand dabei das einzelne Element und seine Funktion(en) für Gesellschaft (z. B. Religion) im Mittelpunkt. Dementsprechend verlangte er als nächsten Schritt die Spezifizierung der Einheiten, für die ein gegebenes soziales oder kulturelles Element funktional ist (vgl. 1967, S. 129), es ging ihm also um die Ausstrahlungskraft eines Elementes. In dieser Arbeit wird vorgeschlagen, alternativ ein einzelnes Element bzw. Objekt bzw. Problem in den Mittelpunkt zu stellen, auf das andere Elemente funktional einwirken, um damit seine Bedingungen der Möglichkeit analysieren zu können. Die Voraussetzung für die Feststellung funktionaler Äquivalenzen ist der Vergleich. Es ist ein komparatistisches Vorgehen und entweder auf der Zeitebene (historische Forschung mit Vergleich verschiedener Phasen) oder auf der Raumebene (Systemvergleich) möglich. Beim Systemvergleich ist jedoch darauf zu achten, dass die speziellen Gegebenheiten der zu vergleichenden Einheiten (und möglichst auch ihre historischen Entstehungsbedingungen) berücksichtigt werden im Sinne eines transkulturellen Vergleichs, der eine Methode der Universal- oder Globalgeschichte ist (vgl. Drews \& Oesterle, 2008). Eine Generalisierung von Strukturen ist unzulässig. Die funktionale Methode ist zusammenfassend: 
- interpretativ,

- reflexiv,

- kontextualisierend,

- rekonstruierend,

- komparativ (über Zeit und/oder Raum).

\section{Anwendungsbeispiel 1: Medienproduktgeschichte}

In der neuen Kulturgeschichte geht es um symbolische Formen, kulturelle Sinnbildungen und Wissensordnungen. Friedrich Jaeger (2004, S. 359) nennt in diesem Zusammenhang die Artikulation, Vermittlung und Repräsentation kulturellen Wissens, das medial transportiert und transformiert wird. Es geht um die „medial vermittelten Repertoires von Verständigung und Austausch (...), die den öffentlich-diskursiven Raum von Gesellschaften prägen“ (ebd., S. 531, vgl. auch Nünning, 2005, S. 167). Neben dem Wandel kommunikativer Strukturen ist der Wandel der „Institutionalität der Medien“ (ebd.), der ästhetischen Repräsentationen, aber auch der technische Medienwandel wichtig. Zudem wird die bedeutungskonstituierende Rolle der Rezipienten anerkannt (vgl. Nünning, 2005, S. 166), die nicht nur Empfänger sind, sondern im Prozess der kulturellen (Re-)Produktion mit den Medien interagieren. Auch die „wechselseitigen Einflüsse von ,hoher ${ }^{`}$ und ,niederer' Kultur" (ebd.) werden beachtet.

Eine solche ästhetische Repräsentation stellt ein Medienprodukt wie eine Zeitschrift, eine Zeitung, ein Fernsehprogramm oder ein Radioprogramm dar. Mit Hilfe einer funktionalen Analyse lässt sich nicht nur der Wandel eines solchen Medienprodukts rekonstruieren, sondern auch die Gründe für diesen Wandel. Im Sinne des Paradigmas eines komplexen dynamischen Systems ist es notwendig, nicht nur das Medienprodukt und das produzierende Medienunternehmen zu betrachten, sondern auch die individuellen Akteure zu berücksichtigen sowie die Umwelt einzubeziehen. Für Medienproduktgeschichte als funktionale Analyse bedeutet dies die Beachtung des jeweiligen Mediensystems (politisches Feld), rechtlicher (juristisches Feld) und gesellschaftlicher (sozio-kulturelles Feld bzw. sozialer Raum) Voraussetzungen sowie wirtschaftlicher (ökonomisches Feld) Bedingungen. Damit wird die kommunikationswissenschaftlich-intradisziplinäre Herausforderung dieses Forschungsprogramms angesprochen. Es muss neben der Kommunikationsgeschichte auf eine Vielzahl von Wissenschaftskonzepten aus verschiedenen SubDisziplinen zurückgegriffen werden: Medienökonomie, Medienrecht, Kommunikationssoziologie, Öffentlichkeitsarbeit und Rezeptionsforschung. Im Fall der hier vorgestellten Studie wurden auch Konzepte der Musikwissenschaft integriert.

Als Beispiel für eine Medienproduktgeschichte wird das Vorgehen bei einer Radioprogrammgeschichte geschildert. Klassik Radio ist ein privates Radioprogramm in Deutschland und stellt in zweifacher Hinsicht eine Besonderheit in der deutschen Radiolandschaft dar. Einerseits war die Verbreitung von (Hoch-)Kultur bis zur Gründung von Klassik Radio in Deutschland exklusiv den öffentlichrechtlichen Sendeanstalten vorbehalten (vgl. Lüthje, 2010a). Andererseits ist Klassik Radio durch die Selbstdefinition als bundesweites Radio mit einem stetig dichter werdenden Netz von terrestrischen Frequenzen eine Ausnahme im von 
lokalen, regionalen und landesweiten Sendern dominierten privaten Radiomarkt in Deutschland. Ebenfalls eine Besonderheit ist die soziokulturelle Relevanz der Programmentwicklung von Klassik Radio, weil „Klassik“ in der deutschen Kultur ein wichtiges Distinktionssysmbol aus dem Bereich der Hochkultur ist. Deutungsmacht über dieses Distinktionssymbol hat traditionell das Bildungsbürgertum. Mit dem kommerziellen Privatsender Klassik Radio wurde dieses Distinktionssymbol quasi profanisiert. Mit dem programmlichen Wandel war auch ein Wandel der Bedeutung dieses Distinktionssymbols verbunden.

Rekonstruiert wurde die Programmgeschichte beginnend mit der Planungsphase (1989) bis zum Zeitpunkt der Datenerhebung (2003). Sendebeginn war im Oktober 1990. Von 1990 bis 2003 konnten drei Entwicklungsphasen nachgewiesen werden, die mit der Organisationsgeschichte des Senders korrespondierten und von jeweils einer Person geprägt wurden. Die Phasen wurden nach den Schlüsselakteuren, aber auch nach den Aussagen der Claims (Hauptslogans) benannt. Der Claim ist die Zusammenfassung der ,Kultur' eines Senders im Sinne von Vorstellungen über sich selbst, also feldspezifisch und habitusgebunden. In ihm bündelt sich die Quintessenz des Formates. Er wird als wichtigster Bestandteil der Eigenpromotion im Programm und der Off-Air-Werbung beständig transportiert. Im Falle von Klassik Radio ist er noch wichtiger, weil sich in ihm die emotionale Vorstellung (Konnotation), die Bedeutung von Klassik und ihrer Wirkung, wie sie der Sender verstand, unmittelbar den Hörern mitteilte. Mit dem Claim wurden also gleichzeitig das Image des Senders und das Image klassischer Musik transportiert. In der Ära Roosen galt der Claim „Ab jetzt macht Klassik Spaß“. Henry C. Brinker etablierte im Dezember 1995 „Musik für gewisse Stunden“ und 1997 „Musik zum Entspannen und Genießen“ und setzte damit auf Sinnlichkeit. Ab 1999 versprach Ulrich Kubak mit „First Class Music“ Luxus. Dementsprechend wurden die Phasen benannt:

- Phase 1: Ära Roosen - Spaß (1990 - 1995);

- Phase 2: Ära Brinker - Sinnlichkeit (1995 - 1999);

- Phase 3: Ära Kubak - Luxus (1999 - 2003 ff.).

Neben den veränderten Claims konnten an Hand der Sendeschemata Unterschiede in der Programmstruktur und in den Inhalten (sowohl Wort als auch Musik) festgestellt werden. Jede Phase wies eine typische Programmierung auf. Aber die einzelnen Phasen waren wiederum in Segmente aufgeteilt, was nicht allein aus den Vorstellungen der Programmverantwortlichen resultieren konnte. Ökonomische Überlegungen (Art der Finanzierung und finanzielle Lage) brachten ebenfalls gravierende Veränderungen.

Elemente der Programmentwicklung von Klassik Radio waren sieben Instanzen und neun Faktoren, die am Ende dieses Kapitels benannt und in einem Funktionsmodell dargestellt werden. Hier soll jedoch schon darauf hingewiesen werden, dass von besonderer Relevanz die gegenseitige funktionale Neutralisierung der beiden wichtigsten Instanzen Landesmedienanstalten und Werbeindustrie war, die sich auf den wichtigsten Faktor Finanzen und darüber auf die Zielgruppendefinition auswirkten. Privates Radio wird in Deutschland hauptsächlich über Werbung finanziert. Die Werbeindustrie besteht auf eine möglichst junge Hörerschaft. 
Um diese zu erreichen ist auch die Zielgruppe des Senders jung, das traditionelle klassische Publikum hingegen ist gehobenen Alters. Dies führte zu einem Wandel des Musikprogramms, um den Geschmack junger Leute zu treffen. Eine Änderung des Sendernamens wurde jedoch durch die föderale Struktur des deutschen Privatradiosystems und die Lizensierungspraxis der Landesmedienanstalten verhindert. Die hohen Lizenz- und Verbreitungskosten erhöhten zudem den Druck, das Programm kostengünstig zu produzieren und beförderten eine Produktionspraxis mit geringem personalen und hohem technischen Aufwand im Kontext der technologischen Entwicklung (computergestützte Programmplanung). Nur durch diese Konstellation ist der starke Bedeutungswandel von Klassik in und mit dem Programm erklärbar. Die performative Vision des Senders ist ein Produkt von Ökonomisierungs- und Legitimationsdiskursen, die sich überschneiden und die in den deutschen gesamtgesellschaftlichen Raum (Mediensystem, politisches Feld, kulturelles Feld, ökonomisches Feld) eingebettet sind. Damit sind strukturelle Rahmenbedingungen gegeben, innerhalb derer die Programmverantwortlichen entsprechend ihrer habituellen Dispositionen das Programm gestalten können.

\subsection{Definition des Forschungsobjekts}

Der erste Schritt bei einer funktionalen Analyse ist die Definition des Forschungsobjektes, im Falle einer Medienproduktgeschichte des Mediums und des Medienprodukts. Für die hier dokumentierte Fallstudie wurde folgender Objektbereich festgelegt:

- Medium: Radio;

- Sender: Klassik Radio;

- Medienprodukt: Radioformat, Gesamtprogramm mit Fokus auf das Musikprogramm;

- Mediensystem, Gesellschaft und Kulturkreis: Deutsch.

Dabei ist es in selbstreflexiver Einstellung nötig zu klären, wie das Interesse an dem Forschungsobjekt entstanden ist. In diesem Fall war dies eine Differenz des gehörten Programms von Klassik Radio zu habituellen Dispositionen der Autorin, die als Musikalienhändlerin, Musikerin und Konzertgängerin mit ,üblicher' Klassik, dem klassischen Kanon und den Praktiken des Konsums ,hoch'-kultureller Produkte vertraut war.

Um eine verlässliche und dichte Rekonstruktion des Programms und seiner Veränderungen zu leisten und die Bedingungen sowie Instanzen und Faktoren für den Wandel nachzuweisen, musste ,archäologisch' auf eine Vielzahl von Quellen differierenden Ursprungs zurückgegriffen werden. Die nächsten Schritte erfolgten nicht in einer linearen Abfolge, sondern simultan. Jedes neue Puzzlestück brachte eine Revision der vorher getroffenen Entscheidungen und Interpretationen mit sich und auch teilweise die Frage nach einer neuen Methode der Datenerhebung. 


\subsection{Erhebungsmethoden}

Die Erhebungsmethoden sind nicht bestimmten Elementen zugeordnet. Jede Quelle brachte Informationen zu verschiedenen Elementen und bestätigte die durch andere Erhebungsmethoden gewonnenen Informationen oder führte zur kritischen Revision von vorherigen Interpretationen. Für die Programmgeschichte von Klassik Radio wurden qualitative und quantitative Methoden der empirischen Sozialforschung (Experteninterview, quantitative Inhaltsanalyse, Sekundäranalyse) und der Geschichtswissenschaften (Rekonstruktion und Quellenkritik) angewendet.

Am Anfang stand die dokumentenanalytische Auswertung der publizierten Materialien zu Klassik Radio:

- Selbstdarstellung auf der Homepage www.klassikradio.de mit detaillierten Informationen zum Ist-Zustand des Senders: Playlist für eine Woche, Sendegebiet, aktuelles Sendeschema, Wortinhalte, Kontaktadressen.

- „Klassik on Air“ (Programmzeitschrift von September 1994 bis Januar 1996): Programm, Werbung und Sponsoren.

- Jahrbücher der Landesmedienanstalten und Hörfunkjahrbücher: Informationen zu Gesellschaftern und Zielgruppen.

- Pressemitteilungen der Landesmedienanstalten auf deren Homepages (bis ca. 1997 zurückdatiert): Lizenzerteilungen und Frequenzerweiterungen.

- Media Analyse (MA): Rezeption.

- Wissenschaftliche Publikationen zu Klassik Radio.

- Zeitungsartikel über Klassik Radio: Kritik und öffentliche Wahrnehmung.

Dann wurde Kontakt sowohl zu der Sendezentrale in Hamburg als auch zu der Muttergesellschaft Euro Media Group in Augsburg (inzwischen umbenannt in ,Klassik Radio AG') aufgenommen. Der Zweck dieses Vorgehens war, Zugang sowohl zu relevanten Personen, als auch zu den relevanten, organisationsinternen, unveröffentlichten Informationen zur Programm- und Organisationsgeschichte von 1989 (Planungsphase) bis zur Datenerhebung im Jahr 2003 zu bekommen. Die relevanten, unternehmensinternen Dokumente wurden quellenkritisch nach Autor, Textsorte, Zeitpunkt und Ort der Erstellung sowie Adressat behandelt:

- Lizenzanträge an und Lizenzerteilungen durch die Landesmedienanstalten,

- darin: Programmschemata, Playlists (Sendelisten), Contents (Inhalte) und Organisationsaufbau,

- Kommunikation mit den Landesmedienanstalten zur Einhaltung regionaler Anteile,

- Interne Kommunikation (Memos, Sitzungsprotokolle) zur Programmplanung,

- darin: Stundenuhren,

- Stylebooks.

Da für das aktuelle Programm zurzeit der Datenerhebung keine detaillierten Planungsunterlagen zugänglich waren, wurde eine quantitative Musikprogrammanalyse anhand der auf der Internetseite www.klassikradio.de veröffentlichten Sendelisten der Woche vom 3. bis 9. Mai 2003 vorgenommen. Die Kategorien wurden 
analog zu den Informationen über die vorangegangenen Phasen definiert, um Vergleichbarkeit herstellen zu können.

Um die aus den schriftlichen Quellen gewonnenen Daten bzw. die Interpretationen zu überprüfen und mit Hintergrundinformationen anzureichern, wurden face-to-face nichtstandardisierte, narrative und situationsflexible Experteninterviews geführt. Doch auch die Aussagen bzw. Erinnerungen der Interviewten konnten durch die anderen Daten überprüft werden. Der Experte ist oder war Teil des zu untersuchenden Handlungsfeldes (in diesem Fall Klassik Radio). Das Wesen einer Programmgeschichte liegt allerdings in der historischen Perspektive begründet, so dass auch ehemalige Mitglieder der Organisation zu ihrer damaligen Funktion interviewt wurden. Die befragten Personen verfügen oder verfügten über problemlösende oder Lösungen mitbestimmende Entscheidungsbefugnisse bzw. über einen privilegierten Zugang zu Informationen, also Insiderwissen. Entsprechend wurde jeder Experte mit für ihn spezifischen Fragestellungen konfrontiert. Biographisches oder Persönliches wurde in den Interviews selbst ausgeklammert, auch um den Interviewten ein ,sicheres Feld' für ihre Aussagen zu bieten. Ihre Dispositionen wurden jedoch anhand der offiziellen Lebensläufe und ihrer Stationen in die Analyse eingearbeitet.

Die Interviews wurden auf Tonband mitgeschnitten und anschließend teil-transkribiert und qualitativ-inhaltsanalytisch ausgewertet. Primäre Zielgruppe der Untersuchung waren Entscheidungsträger: der Initiator Manfred Lahnstein, alle Geschäftsführer und Programmdirektoren zwischen 1990 und 2003; aber auch Personen, die über relevantes Organisationswissen die Produktion des Programms betreffend verfügen: Musikredakteure und Moderatoren. Der Programmdirektor legt zusammen mit der Geschäftsführung das Format, also die grundsätzliche Programmfarbe, fest. Er entwickelt Programmrichtlinien und verschriftlicht und visualisiert diese in Form von Sendeplänen, Programmuhren und Stylebooks/Moderationsleitfäden (vgl. Sturm \& Zirbik, 1996, S. 81). Eine wichtige Aufgabe ist die Kommunikation dieses Ordnungsrahmens an die Mitarbeiter in den Bereichen Musik, Redaktion und Moderation sowie die Kontrolle der Ausführung. Die Fragen an die Programmdirektoren richteten sich gezielt an die Planungs-, Kommunikator- und Kontrollfunktion innerhalb des Senders. Die ehemaligen und derzeitigen Geschäftsführer wurden vor allem zu den Aspekten der Programmpolitik des Senders befragt, da sie damit unmittelbar in die Gestaltung des Radioprogramms eingriffen. Motive, Zielsetzung, Entscheidungsfaktoren und Durchführung wurden fokussiert. Dies beinhaltete auch Aspekte von Macht und Kontrolle, Aspekte des Unterhaltungs- und Kulturverständnisses und Aspekte der Ökonomie. Die Interviews wurden in den privaten oder geschäftlichen Räumen der Befragten, also in ihrem gewohnten Umfeld, durchgeführt.

Der Indikator für den Erfolg eines Radioprogramms in Deutschland ist die im Auftrag der Arbeitsgemeinschaft Media Analyse AG.MA durchgeführte Media Analyse (MA). Die Hörerzahl, die Zusammensetzung der Hörerschaft und die Nutzung von Klassik Radio wurden mit einer Sekundäranalyse der Media Analyse rekonstruiert. Dabei wurde der Wandel der Erhebungsmethoden der Media Analyse berücksichtigt. 


\subsection{Elemente}

Auf Basis der erhobenen Daten wurden Elemente definiert, die eine Veränderung des Programms beeinflussten. Dafür mussten verschiedene Felder berücksichtigt werden:

- Mediensystem: Das deutsche, förderalistisch organisierte, duale System (fix). Klassik Radio ist ein privat-kommerzieller Sender, Kulturprogramme waren bisher Privileg der öffentlich-rechtlichen Sendeanstalten.

- Ökonomisches Feld: Abhängigkeit der privaten Sender von der Werbeindustrie und Alternativen (variabel).

- Medienunternehmen: Organisationsgeschichte, Praxis der Programmproduktion, Habitus der Programmverantwortlichen.

- Publikum: Rezeptionsgeschichte, Praxis der Medienrezeption.

Aus diesen Feldern wurden (ebenfalls nach den erhobenen Daten) sieben Instanzen (als aktive und entscheidende Elemente) bestimmt, die über neun Faktoren (als passive, mitwirkende Umstände) interagierten. Wichtig war es, die subjektiven Motive und Ziele der einzelnen Instanzen (auch Gruppen oder Einzelpersonen) mit zu erheben, um diese mit den Folgen abzugleichen:

- Instanzen: Rezipienten, Landesmedienanstalten (LMA), Sponsoren, Plattenlabels, Werbewirtschaft, Eigentümer, Programmverantwortliche.

- Faktoren: Vermarktungsstrategie, Finanzen, Verbreitung, medienrechtliche Anforderungen, Image, Zielgruppendefinition, Personal/Technik, Mediengesetze, Habitus (subjektiv-persönliche Vorstellungen bzw. habituelle Dispositionen der Programmverantwortlichen).

\subsection{Zusammenführung}

Die Zusammenführung der Daten oder der Ereignisse erfolgt in einer Partitur. Die Daten werden in Zeitachsen eingetragen, man könnte von einer reinen Zeitreihenanalyse sprechen. Jedoch werden hier die Zeitachsen übereinandergeschichtet und interpretiert. Die Daten können regelmäßig anfallen (wie z. B. die Veröffentlichung der Ergebnisse der halbjährlichen Media Analyse) und sind damit in ihrem Erscheinen berechenbar. Sie können jedoch auch unregelmäßig anfallen wie z. B. Wechsel auf der personalen Ebene oder Programmrelaunches. In der funktionalen Analyse geht es darum, diese Ereignisse in Verbindung zu bringen. Ihre zeitliche Nähe ist ein wichtiger Indikator für ein Zusammenspiel, sagt aber nicht zwingend etwas über ihren Zusammenhang oder die Stärke und Qualität des Einflusses aus. In der funktionalen Analyse stehen die statistischen Zusammenhänge nicht im Vordergrund. Die Verbindung der Ereignisse auf den verschiedenen Ebenen mit Blick auf ihren Einfluss auf die Veränderung des fokussierten Objektes (in diesem Fall des Programms von Klassik Radio und der Bedeutung von Klassik) ist auf Interpretation gegründet und wird durch die dichte Datensammlung, Reflexivität (bzw. den Diskussionen in einem Forscherteam) und auch eine gewisse Wiederholung von Abfolgen im Zeitverlauf größtmöglich abgesichert. Zur Veranschaulichung eine stark vereinfachte Darstellung: 
Abbildung 2: Partitur

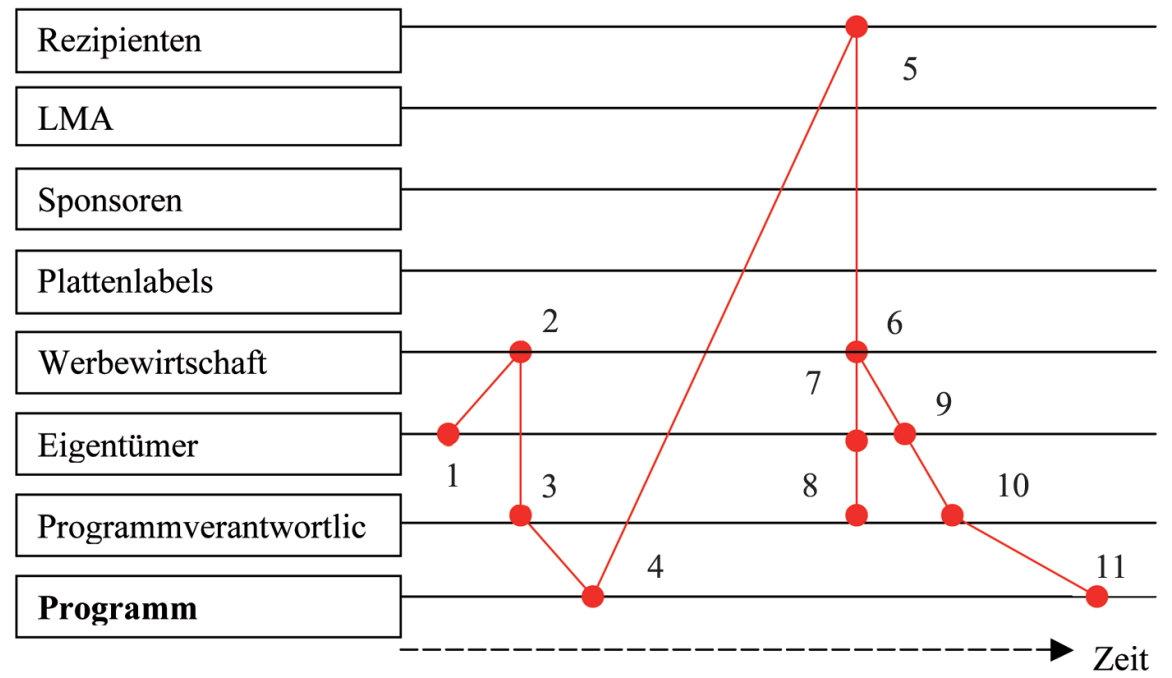

Die Eigentümer (1) treffen an einem bestimmten Zeitpunkt eine Entscheidung für eine Finanzierungsform: Das Programm soll über Spotwerbung finanziert werden. Die Werbewirtschaft (2) wird kontaktiert und kommuniziert ihre Anforderungen an das Programm an die Programmverantwortlichen (3), die diese im Rahmen ihrer habituellen Dispositionen bei der Konzeption des Radioprogramms (4) berücksichtigen. Mit einem gewissen zeitlichen Abstand werden (5) die Nutzungsdaten in der Media Analyse veröffentlicht, die zeitgleich von Werbewirtschaft (6), Eigentümern (7) und Programmverantwortlichen (8) wahrgenommen werden. Die Werbewirtschaft signalisiert an die Eigentümer (9) über den Ankauf von Werbezeiten oder auch in indirekter Kommunikation über Vertreter Zufriedenheit oder Unzufriedenheit mit den Hörerzahlen und der Zusammensetzung der Hörerschaft. Die Eigentümer geben Anweisungen an die Programmverantwortlichen (10), die das Programm dementsprechend und im Zusammenspiel mit ihrer eigenen (habituell gebundenen) Wahrnehmung umgestalten. Mit einem gewissen zeitlichen Abstand sind die Auswirkungen im aufgeschalteten Programm (11) festzustellen.

Die tatsächlichen Prozesse sind sehr viel komplexer, die Programmverantwortlichen sind z. B. bei der Planung und Realisierung des Programms auch von den Vorgaben der Landesmedienanstalten abhängig, die einen permanenten Einfluss haben, auch wenn auf den Zeitreihen keine besonderen Ereignisse eingetragen sind. Verschiedene Prozesse laufen zeitgleich ab. Aus den Zeitachsen und den qualitativen Informationen zu den Daten (also aus den gesammelten Informationen heraus und nicht im Vorfeld) lassen sich Indikatoren für verschiedene Phasen ableiten, in die sich die Programmgeschichte einteilen lässt. Im Vergleich der verschiedenen Phasen können funktionale Äquivalente festgestellt werden. Als Indizien für Zäsuren bei Klassik Radio wurden eine veränderte Programmstruktur, veränderte Programminhalte (Wort und Musik) und ein neuer Claim (Hauptslogan) gewertet. Als innersystemische Instanzen für die Phaseneinteilung wurden 
Geschäftsführung und Programmleitung angesehen. Mit den aus den verschiedenen Quellen gewonnenen Informationen konnten eine Planungsphase (1989) und die drei Entwicklungsphasen von 1990 bis 2003 bestimmt werden.

Wenn diese Partitur mit ihren interpretierten Verknüpfungen , auf den Kopf gestellt' und auseinandergezogen wird, wenn man also in eine ,Röhre' blickt, können in einem Funktionsmodell die Verknüpfungen der verschiedenen Instanzen quasi entzeitlicht und auf generelle Prozesse reduziert visualisiert werden. In der Abbildung sind die interdependenten Instanzen und Faktoren für das Programm von Klassik Radio dargestellt.

\section{Abbildung 3: Funktionsmodell}

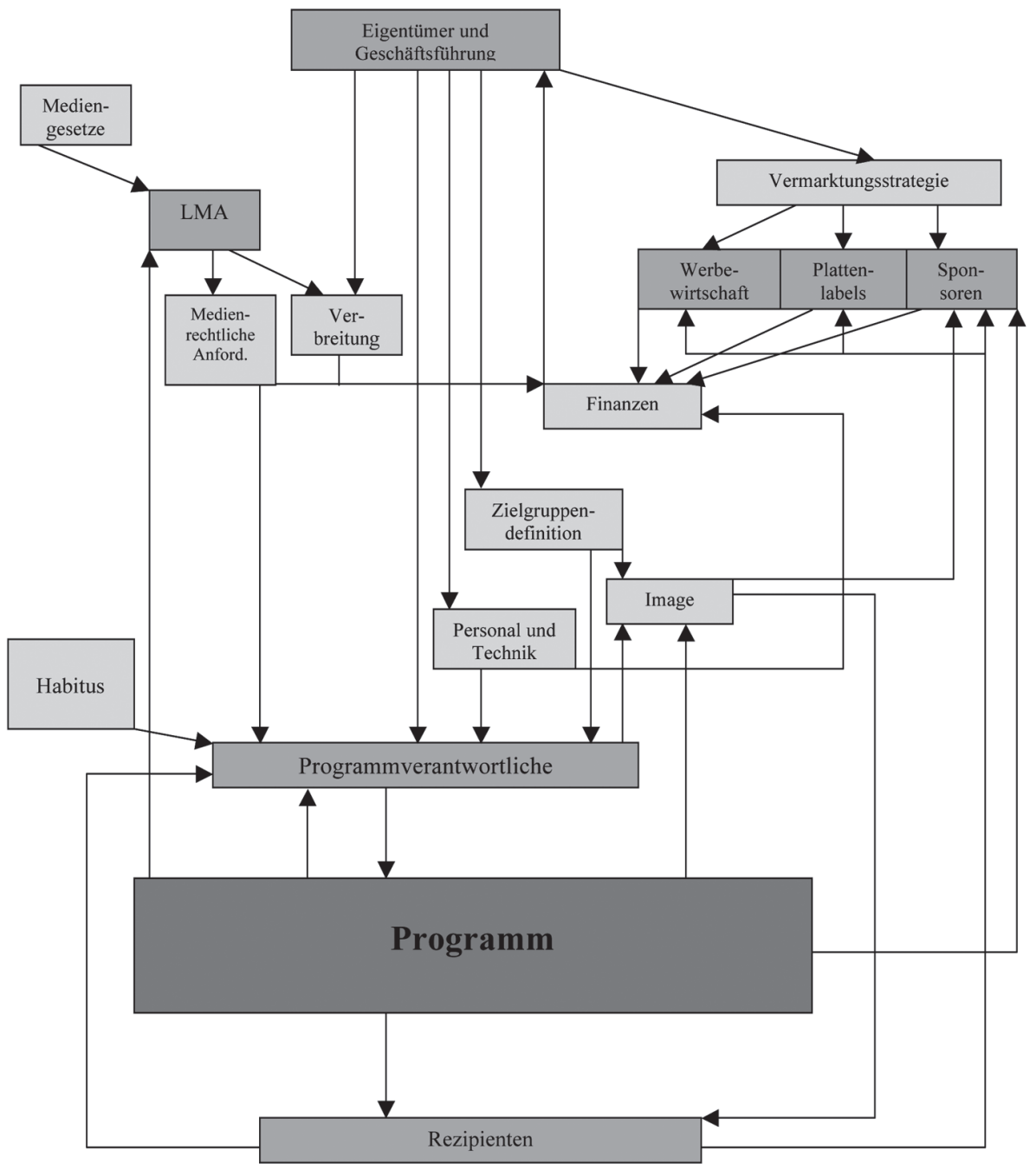


Auf den ersten Blick erscheint dieses Funktionsmodell wie ein Schaltplan. Dieser Eindruck ist jedoch zu eindimensional. Mit dem Modell werden die komplexen Wechselwirkungen der verschiedenen Instanzen und Faktoren visualisiert, deren Qualität einer detaillierten und deskriptiven Erläuterung bedarf, die aber nicht mehr Gegenstand dieses Aufsatzes sein kann.

Das erste Anwendungsbeispiel für eine funktionale Analyse betraf eine originär kommunikationswissenschaftliche Fragestellung. Klassik Radio als Forschungsprojekt war klein genug und der Zeitraum von 14 Jahren überschaubar genug, um von einer Person bearbeitet zu werden, jedoch kann man hier schon von einem Grenzwert sprechen. Noch komplexere Kulturgeschichten als funktionale Analysen würden die Kapazitäten von Einzelpersonen sprengen. Hier ist also intradisziplinäre Teamarbeit gefragt. Bei der Programmgeschichte von Klassik Radio wurde vor der Begriffsbildung mit der Datenerhebung begonnen. Gleichwohl muss hier noch einmal betont werden, dass Theorieformulierung und Datenerhebung danach parallel verliefen und es im Forschungsprozess immer wieder zu Reformulierungen und Umjustierungen sowohl bei der Empirie als auch bei der Theorie kam.

\section{Anwendungsbeispiel 2: Soziale Erinnerung in der Hazardkommunikation}

Erinnerungsforschung ist ein wichtiger Bereich der neuen Kulturgeschichte. In dem zweiten Anwendungsbeispiel wird das Vorgehen bei einer interdisziplinären funktionalen Analyse mit kommunikationswissenschaftlichen Anteilen zur Genese und Transformation des sozialen Gedächtnisses bei einem regionalen Geohazard dargestellt.

Wie Gesellschaften mit Bedrohungssituationen umgehen, ist perspektivisch gebunden. Aus der Risikosoziologie von Niklas Luhmann (2003) ist die Dichotomie von Gefahr und Risiko bekannt. Eine Gefahr ist eine Bedrohung, die von einem Individuum oder einer Gesellschaft nicht gewusst wird. Mit Eintreten eines Schadensereignisses wird die grundsätzliche Bedrohung bewusst. Wenn davon ausgegangen wird, dass sich das Schadensereignis wiederholen könnte, führt das zur Abschätzung bzw. Berechnung der Wahrscheinlichkeit und des Gefährdungspotentials, das Ereignis wird als Risiko antizipiert. Auf der Basis dieser Antizipation werden Gegenmaßnahmen ergriffen, worauf sich wieder ein Sicherheitsgefühl einstellt. Das Risikokonzept erscheint angesichts der stetig steigenden Zahl von katastrophalen Ereignissen und stetig steigenden Schadenshöhen für Akteure in Politik, Versicherungswirtschaft und Wissenschaft zunehmend als unzureichend. Es wird seit einigen Jahren nach einem alternativen Bewertungs- und Umgangsschema mit Bedrohungen gesucht. Anregungen dafür kommen einerseits aus dem englischsprachigen Raum, aus dem der Begriff des „Hazard“ entlehnt wurde. So hat das Bundesinstitut für Risikobewertung eine „Evaluierung der Kommunikation über die Unterschiede zwischen ,risk' und ,hazard' veröffentlicht (Ulbig, Hertel, \& Böl, 2009). Diesem Bericht vorhergegangen ist die Entwicklung des Hazard- und Resilienzkonzepts in den Umweltwissenschaften. Das Hazardkonzept befindet sich zwischen dem Konzept der Gefahr und dem Konzept des Risikos (Petak \& Atkisson, 1982). Schlüsselbegriffe des Hazardkonzepts sind Resilienz (Wider- 
standsfähigkeit und Fähigkeit zur Selbst-Reorganisation), Vulnerabilität (Verwundbarkeit), Nachhaltigkeit und Adaption (Anpassung). Bei einem Hazard werden Systeme als offen angesehen. Sie interagieren. Durch diese Interaktion wird der Hazard selbst zu einem komplexen dynamischen System. Bei einem technischen Hazard handelt es sich um Mensch-Technik-Interaktion, bei einem Terrorhazard um Interkation gesellschaftlicher (Sub-)Systeme und bei einem Geohazard (als Teil der Umwelt) um Mensch-Natur-Interaktion. Unter einem regionalen Geohazard wird eine durch physisch-geographische Ereignisse und Umstände gegebene Bedrohung der Gesellschaft in einer bestimmten Region verstanden. Wichtige Komponenten des Hazard-Konzepts sind das Bewusstsein der Bedrohung und die soziale Erinnerung an ein traumatisches Schlüsselereignis. Neben der regionalen Betroffenheit von akuten Auswirkungen und latenter Bedrohung hat ein regionaler Geohazard also folgende Merkmale (Neverla, 2009; Ratter, 2009):

- Physisch-geographische Ereignisse und Umstände in einer bestimmten Region/einem Ausschnitt aus der Erdoberfläche;

- nichtlineare Mensch/Natur-Interaktion;

- Unsicherheit und schlechte Vorhersagbarkeit aufgrund von Struktur- und Verhaltenskomplexität;

- Schlüsselereignis: Ein traumatisches Erlebnis und die kollektive Erinnerung daran.

Im zweiten Anwendungsbeispiel für eine funktionale Analyse wird das Vorgehen in dem interdisziplinären StarG-Projekt („Sturmfluten als regionale Geohazards“) vorgestellt. Dieses Projekt wird seit Juli 2009 am Klimaforschungsexzellenzcluster CliSAP an der Universität Hamburg unter der Leitung von Irene Neverla (Kommunikationswissenschaft) und Beate Ratter (Geographie) durchgeführt. Es handelt sich um ein fächergruppenübergreifendes Projekt zwischen Natur-, Gesellschafts- und Kulturwissenschaften und besteht aus vier integrierten Teilprojekten. Die Autorin ist inhaltliche Koordinatorin des Gesamtprojekts. Kernkonzept eines Hazards ist die soziale Erinnerung an ein Schlüsselereignis. Soziale Erinnerung ist kommunikativ konstruiert (vgl. Welzer, 2001). Das StarG-Projekt geht am Beispiel der Sturmflut in Hamburg 1962 der Frage nach, wie die soziale Erinnerung an ein traumatisches Schlüsselereignis aufrechterhalten wird, welche Akteure daran beteiligt sind und wie sich die Erinnerung im Laufe der Jahre verändert. Der kommunikationswissenschaftliche Anteil im Gesamtprojekt bezieht sich auf kommunikative Konstruktionsprozesse und die Funktion des Journalismus bei der Bildung der sozialen Erinnerung. Die Einbindung in die Klimaforschung brachte eine Berücksichtigung der Klimawandelproblematik in der Untersuchung mit sich. Das Projekt ist explorativ und die Modellentwicklung auf Adaption auf Geohazards in anderen Regionen angelegt.

\subsection{Theoriearbeit 1: Morphologische Analyse und Begriffsdefinition}

Interdisziplinäre, integrierte Projekte sind von besonderen Herausforderungen bestimmt. Es ist vor allem notwendig, eine gemeinsame Projektsprache und Projektkonventionen zu entwickeln, die der Terminologie und den Praktiken in den 
beteiligten Disziplinen durchaus widersprechen können. Zudem ist integrative Forschung von einem grundsätzlichen Eklektizismus geprägt. Identifikationsebene ist immer das jeweilige Projekt, nicht die disziplinäre Heimat. Die Entwicklung der gemeinsamen Projektsprache ist ein heikles Projekt informeller interner Wissenschaftskommunikation, denn (abgesehen von der vorausgesetzten Bereitschaft der beteiligten Personen, an diesem Projekt konstruktiv mitzuwirken) ist jeder Wissenschaftler und jede Wissenschaftlerin habituell mit der eigenen Disziplin verstrickt. Neben dem Willen zur Kooperation ist also ein hoher Grad an Reflexionsfähigkeit und die Bereitschaft, eigene disziplinäre Vorstellungen zu relativieren gefragt.

Um die Projektintegration zu befördern empfiehlt es sich, die funktionale Analyse nicht mit Datensammlung zu beginnen, sondern mit Theoriearbeit. Diese integrative Theoriearbeit muss systematisch und moderiert erfolgen. Es bietet sich dafür die morphologische Methode nach Fritz Zwicky (1969) an. Der Begriff Morphologie geht auf Johann Wolfgang von Goethe zurück und bezeichnet Formenlehre. In einer morphologischen Matrix werden verschiedene Lösungen eines Problems mit ihren Merkmalen gegenübergestellt und damit visualisiert. In einem Projektteam können mit Hilfe der gemeinsamen Anwendung der morphologischen Methode die projektinternen theoretischen Konzepte und Begriffe ausgehandelt werden. Die Schriftform der Matrix garantiert die Verbindlichkeit der Übereinkunft. Als Beispiel soll die morphologische Matrix der Bedrohungskonzepte Gefahr, Hazard und Risiko dienen:

\section{Abbildung 4: Morphologische Matrix von Bedrohungskonzepten (Gefahr, Hazard, Risiko)}

\begin{tabular}{|c|c|c|c|c|c|c|}
\hline Bedrohung & Verursachung & $\begin{array}{l}\text { Freiwilligkeit } \\
\text { der Bedrohungs- } \\
\text { situation }\end{array}$ & $\begin{array}{l}\text { Schlüsselereignis } \\
\text { (Erfahrung) } \\
\text { + Erinnerung }\end{array}$ & Wissen & $\begin{array}{l}\text { Sicherheits- } \\
\text { gefühl }\end{array}$ & Handlungsoptionen \\
\hline $\begin{array}{l}\text { Gefahr } \\
\rightarrow \text { Bedrohung } \\
\text { situationsinhärent und } \\
\text { nicht bewusst }\end{array}$ & $\begin{array}{l}\text { System-extern } \\
\text { verursacht } \\
\text { Sichtweise: } \\
\text { geschlossene } \\
\text { Systeme } \\
\end{array}$ & $\begin{array}{l}\text { Unfreiwillig } \\
\text { (durch Nichtwis- } \\
\text { sen keine Wahl) }\end{array}$ & Nein & $\begin{array}{l}\text { Nicht- } \\
\text { gewusstes } \\
\text { Nichtwissen }\end{array}$ & $\begin{array}{l}\text { Sicher: } \\
\text { Bedrohung } \\
\text { Unvorstellbar }\end{array}$ & $\begin{array}{l}\text { Flexibel (kein festes } \\
\text { Handlungsrepertoire } \\
\text { durch Nichtwissen) }\end{array}$ \\
\hline $\begin{array}{l}\text { Hazard } \\
\rightarrow \text { Bedrohung } \\
\text { situationsinhärent und } \\
\text { bewusst } \\
\rightarrow \text { zugehörig: Resilienz, } \\
\text { Vulnerabilität, } \\
\text { Nachhaltigkeit, } \\
\text { Adaption, }\end{array}$ & $\begin{array}{l}\text { Interaktion } \\
\text { zwischen } \\
\text { Systemen } \\
\text { (Geohazard: } \\
\text { Mensch-Natur- } \\
\text { Interaktion) } \\
\text { Sichtweise: } \\
\text { offenes, adaptives, } \\
\text { komplexes, } \\
\text { dynamisches } \\
\text { System }\end{array}$ & $\begin{array}{l}\text { Freiwillig (durch } \\
\text { Wissen: Wahl- } \\
\text { möglichkeit, } \\
\text { Abwägung } \\
\text { Kosten und } \\
\text { Nutzen) }\end{array}$ & $\begin{array}{l}\text { Ja } \\
\text { Erinnerung wird } \\
\text { aufrechterhalten }\end{array}$ & $\begin{array}{l}\text { Gewusstes } \\
\text { Nichtwissen, } \\
\text { Bewusstsein } \\
\text { für die } \\
\text { Vulnerabilität }\end{array}$ & $\begin{array}{l}\text { Unsicher: } \\
\text { eingestellt auf } \\
\text { Überraschungen, } \\
\text { Abwägung von } \\
\text { Kosten und } \\
\text { Nutzen } \\
\rightarrow \text { Bewusstes } \\
\text { Leben mit der } \\
\text { Bedrohung, } \\
\text { Einbau der } \\
\text { Bedrohung in das } \\
\text { Leben }\end{array}$ & $\begin{array}{l}\text { Flexibel (Adaption, } \\
\text { Bewusstsein für } \\
\text { Unsicherheit: } \\
\text { eingestellt auf } \\
\text { radikale } \\
\text { Überraschungen) }\end{array}$ \\
\hline $\begin{array}{l}\text { Risiko } \\
\rightarrow \text { die Möglichkeit des } \\
\text { Eintritts eines } \\
\text { Schadensereignisses: } \\
\text { Wahrscheinlichkeits- } \\
\text { Konstruktion aus } \\
\text { Eintreten und } \\
\text { Schadenshöhe } \\
\rightarrow \text { zugehörig: } \\
\text { Antizipation, Mitigation }\end{array}$ & $\begin{array}{l}\text { System-intern } \\
\text { verursacht (selbst } \\
\text { verursacht) } \\
\text { Sichtweise: } \\
\text { geschlossenes } \\
\text { System }\end{array}$ & $\begin{array}{l}\text { Eher unfreiwillig } \\
\text { (Bedrohung wird } \\
\text { als gemeistert } \\
\text { angesehen, } \\
\text { deshalb } \\
\text { Entscheidung für } \\
\text { das Handeln) }\end{array}$ & $\begin{array}{l}\text { Ja } \\
\text { Nach } \\
\text { Entwicklung von } \\
\text { (scheinbar) } \\
\text { wirksamen } \\
\text { Schutzmaß- } \\
\text { nahmen: } \\
\text { Erinnerung wird } \\
\text { unwichtig }\end{array}$ & $\begin{array}{l}\text { Ignoranz [des } \\
\text { Nichtwissens } \\
\text { und der } \\
\text { Vulnerabilität] }\end{array}$ & $\begin{array}{l}\text { Sicher: Kontrolle } \\
\text { der Bedrohung } \\
\text { durch } \\
\text { Antizipation und } \\
\text { Entwicklung von } \\
\text { Maßnahmen/ } \\
\text { Mitigation } \\
\rightarrow \text { Bedrohung } \\
\text { gemeistert }\end{array}$ & $\begin{array}{l}\text { Unflexibel } \\
\text { (Antizipation }+ \\
\text { Mitigation } \rightarrow \text { festes } \\
\text { Handlungsreper- } \\
\text { toire) }\end{array}$ \\
\hline
\end{tabular}




\subsection{Theoriearbeit 2: Ausarbeitung einer Vortheorie}

Auf der Basis der gemeinsamen Projektsprache werden theoretische Konzepte der beteiligten Disziplinen in das Projekt eingebracht und eine vorläufige Theorie formuliert, mit Hilfe derer ein erstes Funktionsmodell erstellt werden kann. Dieses Modell hat die Aufgabe einer Heuristik, die dazu dient, die integrierten empirischen Teilstudien zu konzipieren.

Im Rahmen des StarG-Projekts wurde die dreidimensionale Anlage des Konzepts eines regionalen Geohazards ausgearbeitet: Raum/Region, soziokulturelle Sphäre und Zeit.

Ein regionaler Geohazard hat zunächst einen besonderen Raumbezug durch (a) die lokalen/regionalen und akuten Auswirkungen und (b) durch die spezifischen geographischen Gegebenheiten. Dieser Raumbezug ist Domäne der beteiligten Geowissenschaften. Zur Sturmflut in Hamburg liegt eine Fülle von Daten vor, die die Entwicklung der Stärke und Häufigkeit dieser Extremereignisse sowie der geophysikalischen Gründe dafür dokumentieren. Auf diese Daten wurde im StarGProjekt zurückgegriffen.

Weiterhin hat ein regionaler Geohazard einen spezifischen soziokulturellen Be$z u g$. Hier sind die Sozial- und Kulturwissenschaften gefragt auf der Basis von Bourdieus Strukturkonstruktivismus. In den Hazard involviert sind gesellschaftliche Agenten (Gruppen und/oder Einzelpersonen), die in ihren unterschiedlichen sozialen Feldern verankert sind: Medien, Interessengruppen (zu denen als gegensätzliche Pole Wirtschaft und NGOs gezählt werden), Wissenschaft, Politik und Bürger. Die Akteure haben sich den jeweils feldspezifischen Habitus und entsprechende Praktiken angeeignet. Die sozialen Felder befinden sich innerhalb des gesamten gesellschaftlichen Raums, der einen gemeinsamen soziokulturellen Rahmen gibt. Zum Hazardmanagement gehört einerseits Mitigation von potentiellen Schäden durch strukturelle Maßnahmen (z. B. Deichbau) und andererseits Adaption an die Bedrohungssituation durch nicht-strukturelle Maßnahmen, die auch Alltagspraktiken beinhalten wie Wissen um das richtige Verhalten bei Alarm. Unter Berücksichtigung der unterschiedlichen Kompetenzen und Interessen der differierenden Felder sind die Aufgaben und Funktionen ausdifferenziert. Politik, Interessengruppen und Bürger erwarten z. B. von der Wissenschaft, Begründungszusammenhänge der Extremereignisse zu erforschen, Problemlösungen zu entwickeln sowie Warnsysteme zur Verfügung zu stellen, mit denen Gefährdungen so früh und so genau wie möglich gemeldet werden können. Die Wirtschaft im Gegenzug ist für Finanzierungen zuständig, stellt aber auch bestimmte Anforderungen an die Politik (ein aktuelles Beispiel ist die Elbvertiefung in Hamburg). Das politische Feld soll Probleme lösen, Entscheidungen treffen, planen und kommunale Strukturen schaffen (welche wiederum hauptsächlich vom wirtschaftlichen Feld und Steuern finanziert werden). Die Gesellschaft, womit in diesem Fall die Summe der verschiedenen sozialen Gruppen und Personen/Bürger gemeint ist, lebt mit dem Hazard. Der Hazard ist Teil des kollektiven Habitus und beeinflusst so Alltagspraktiken und langfristige Entscheidungen (z. B. Handeln nach Alarm; Mitgliedschaft in einer Organisation wie THW, freiwillige Feuerwehr, Deichverband; Entscheidung an welchem Ort und wie ein Haus gebaut wird etc.). Massen- 
medien sind für Warnungen im Ernstfall zuständig. Außerdem haben sie die Aufgaben, über Gefahren zu informieren, wissenschaftliche und politische Lösungen bzw. Entscheidungen zu veröffentlichen (und sie kritisch zu hinterfragen) sowie über Ausmaß und Art der Schäden, Gründe und Konsequenzen zu berichten. Dies alles sind intendierte Funktionen.

Individuelle Akteure haben jedoch auch einen eigenen Habitus. Individuen bewegen sich relativ frei zwischen den unterschiedlichen sozialen Feldern innerhalb einer soziokulturellen Sphäre. Die Interaktion von Individuum und Struktur führt zu einem permanenten sozialen und kulturellen Wandel. Gleichzeitig ist die soziokulturelle Sphäre nicht nur Objekt des Wandels, sondern auch historisch gewachsen und in Traditionen verwurzelt.

Raum und soziokulturelle Sphäre können nicht getrennt betrachtet werden, sie sind interdependent. In anderen Worten: Die Umwelt (und damit das Ausmaß des regionalen Geohazards) ist das Ergebnis der oben beschriebenen zirkulierenden und nichtlinearen Mensch-Natur-Interaktion. Traditionen und historisch gewachsene soziokulturelle Sphäre ebenso wie individuelle Konzepte haben sich in Abhängigkeit zu naturräumlichen Rahmenbedingungen entwickelt und beeinflussen ihrerseits die Natur. Sie sind also nicht determiniert sondern interdependent. Zum Verständnis dieser Interdependez soll das Beispiel Hamburg beitragen. Die Stadt konnte sich nur aufgrund ihrer geographischen Lage am Ende des Elbeästuars zu dem heutigen Zustand entwickeln. Hamburg, wie wir es kennen, wäre in den Alpen nicht möglich. In früheren Zeiten war es ein Standortvorteil, Waren auf dem Wasserweg weit in das Land herein transportieren zu können. Die technologische Entwicklung hat dazu geführt, dass heutige Containerschiffe eine Größe erreicht haben, die das Anlaufen des Hamburger Hafens erschwert. Die (Hafen-)Wirtschaft fordert, die Elbe auszubaggern. Damit werden die Entstehungsbedingungen von Sturmfluten verändert. Aufgabe des politischen Feldes ist es, nach Abwägung der verschiedenen Interessen und potentiellen Auswirkungen, eine Entscheidung für oder wider Elbvertiefung zu treffen.

Auf der Basis dieser theoretischen Vorarbeit wurde ein erstes Funktionsmodell entwickelt, in dem die Interdependez der verschiedenen Systemelemente visualisiert wird: 


\section{Abbildung 5: Regionaler Geohazard: Interdependenz von Elementen}

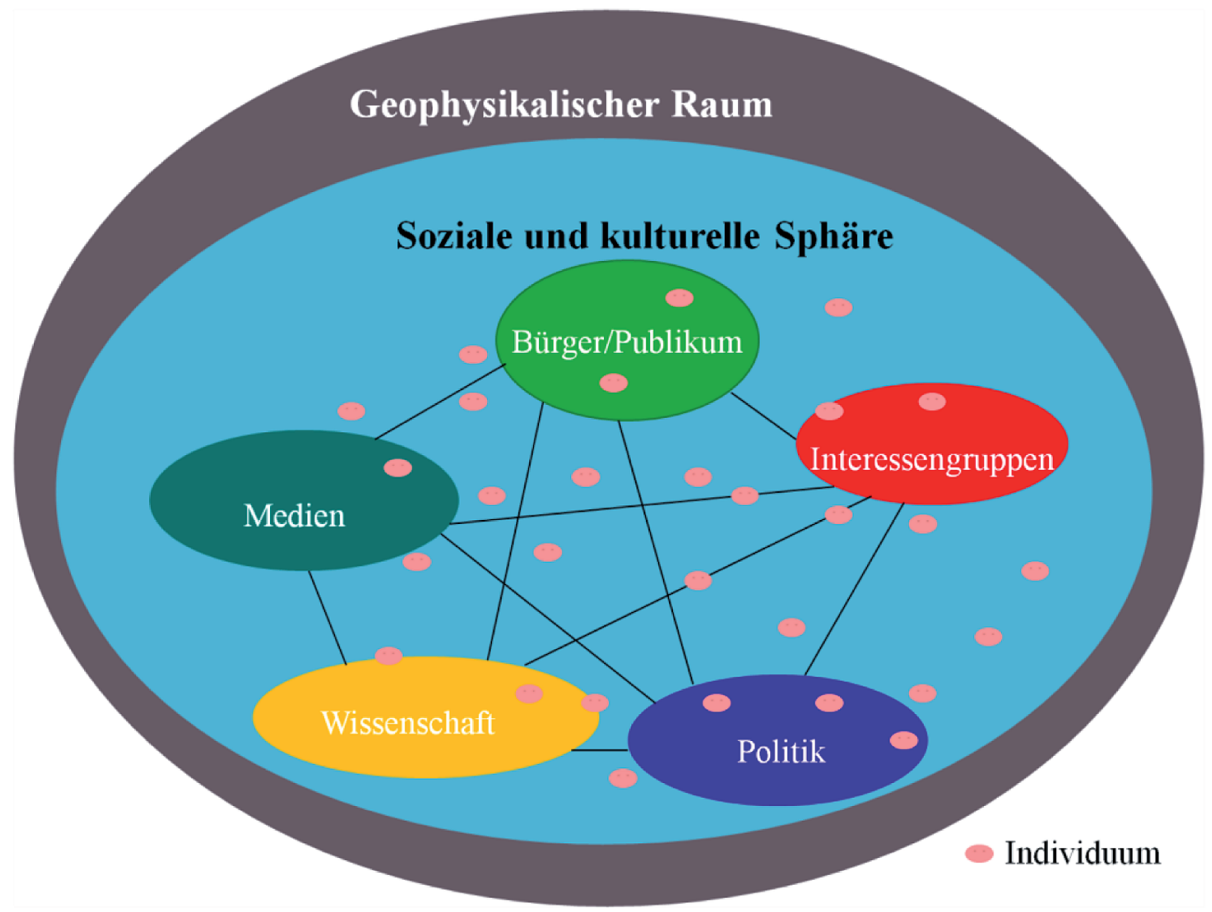

Als dritte Dimension hat ein regionaler Geohazards einen bestimmten Zeitbezug, der sich (a) über die Erinnerung an das traumatische Ereignis an die Vergangenheit richtet, (b) durch die Antizipation zukünftiger Ereignisse in die Zukunft denkt und (c) in der Gegenwart im Bewusstsein präsent ist und zur Änderung von Praktiken führt.

\subsection{Soziale Erinnerung und Hazardkommunikation: Heuristisches Modell}

Für die Planung einer empirischen Untersuchung von Hazardkommunikation ist es wichtig eine Vorstellung davon zu haben, wie soziale Erinnerung an das Schlüsselereignis präsent bleibt und wie sie sich wandelt (und mit ihr die Wahrnehmung des Gefahrenpotentials). Startpunkt ist immer ein traumatisches Schlüsselereignis, dem zum Zeitpunkt seines Geschehens gesellschaftlich eine hohe Relevanz zugeschrieben wird. Dessen soziale Erinnerungskarriere wird durch das komplexe $\mathrm{Zu}-$ sammenspiel von Akteuren und Agenten in den Dimensionen Zeit, Raum und soziokulturelle Sphäre bestimmt. Eine besondere Rolle spielen dabei die tagesaktuellen Medien bzw. der Journalismus. Dies ist der kommunikationswissenschaftliche Anteil am Gesamtprojekt.

Für die Bildung gesellschaftlicher Erinnerung ist das Zusammenspiel von tagesaktuellen Medien (respektive Journalismus) und Medienrezpienten, deren Wahrnehmung der medialen Erinnerung und ihre Weiterschreibung der Erinnerung im 
Kontext ihres sozialen Umfeldes über Anschlüsse wichtig. Nach Zierold (vgl. 2006, S. 151) werden vergangene Ereignisse in der Gegenwart nur dann erinnert, also re-aktualisiert, wenn sie in der Gegenwart relevant gemacht werden. Die Relevanzzuweisung in der Gegenwart erfolgt dabei in zwei Schritten: erstens die mediale Herstellung eines Erinnerungsanlasses und zweitens dessen soziale Akzeptanz durch Erinnerungsanschlüsse. Diese Anschlüsse können sowohl kurzfristig Kommunikation und bewusste Handlungen als auch mittel- bis langfristig habitualisierte Alltagspraktiken sein. Tagesaktuelle Medien und Journalisten sind als „Memory-Agents“ (Zelizer, 2008) für „Erinnerungskarrieren“ (Zierold, 2006) zuständig. Sie sind „Erinnerungs- und Gedächtnisgeneratoren“ (Reinhard \& Jäckel, 2005, S. 102) über die Selektion von historischen Ereignissen, die in der Berichterstattung berücksichtigt werden. Sie setzen die „Erinnerungs-Agenda“ und folgen dabei Nachrichtenfaktoren. Medien sind immer Bestandteil des nationalen Mediensystems sowie des kulturellen und gesellschaftlichen Systems, das sich innerhalb eines klar bestimmbaren geographischen Raums befindet. Die Akteure in den Medien handeln in feldspezifischen habitualisierten Praktiken. Dazu gehören die berufspraktischen Routinen der Nachrichtenselektion und -aufbereitung. André Donk (2009) unterscheidet drei spezifische Arten der journalistischen Berichterstattung über Vergangenheit für die Herstellung von Erinnerungsanlässen: Jahrestagsjournalismus, historische Kontextualisierung und historische Analogien. $\mathrm{Zu}$ Jahrestagen wird an bedeutsame Ereignisse erinnert (Robinson, 2009). Bei historischen Analogien werden historische Ereignisse für ähnliche Ereignisse ohne direkte Betroffenheit der Rezipienten zur Illustration und zur Herstellung von kultureller und sozialer Nähe zitiert (z. B. Flutkatastrophen im Ausland). Historische Kontextualisierung erfolgt beim Eintreten eines dem historischen Ereignis ähnlichen Folgeereignisses (z. B. folgende Sturmfluten in Hamburg). Das historische Ereignis wird in allen drei Fällen zur Erklärung herangezogen und seine Geschichte wird medial fortgeschrieben.

Gesellschaftliche Erinnerungen sind kontingent. Tagesaktuelle Medien sind nicht nur Generatoren, sondern auch Erinnerungs- und Gedächtnistransformatoren. Das historische Ereignis wird zu den Erinnerungsanlässen aus dem Fundus des sozialen Gedächtnisses geholt und in einem Akt der Erinnerung aktualisiert, dem gegenwärtigen Rahmen angepasst. Erinnerung (auch soziale) ist nicht primär vergangenheitsbezogen, sondern ein „Prozess in der Gegenwart“ (Zierold, 2006). Transformierende Ereignisse am Beispiel der Berichterstattung über die Sturmflutgefahr in Hamburg sind der globale Diskurs um den Klimawandel und dessen Zusammenhang mit Naturkatastrophen sowie neue Projekte der Stadtplanung wie die HafenCity, die in einem besonders von Sturmfluten bedrohten Gebiet gebaut wird.

Nun wird die Rezeptionsdimension in das Modell eingeführt. Rezipienten übernehmen die medial präsentierte Erinnerung nicht exakt. Medien und Publikum interagieren im Konstruktionsprozess der sozialen Erinnerung. Soziales Gedächtnis ist nach Welzer (2001) das „Universum einer Vergangenheitsbildung en passant ", die überwiegend unbewusst und absichtslos passiert. Die medialen Erinnerungsereignisse initiieren Anschlusshandlungen, speziell Gespräche in Familien oder anderen sozialen Gruppen mit Bezug auf eigene Erfahrungen und die eigene 
Geschichte, Regionalgeschichte und auch Mythen. Ein wichtiges Medium der sozialen Praxis der Vergangenheitsbildung (Welzer, 2001) ist „Memory Talk“. Diese Konversationspraxis verknüpft und vermischt Traditionen, eigene frühere und gegenwärtige Erfahrungen und die mediale Erinnerungsarbeit. Das Ergebnis ist eine Erinnerung: eine Wahrheit, relativ zu Zeit, Raum, sozialen Bedingungen und individueller Wahrnehmung, aber nicht zweifelsfrei identisch mit der historischen Realität.

Auf der Basis dieser theoretischen Vorarbeit kann die zeitliche Komponente in das Funktionsmodell (vgl. Abb. 5) eingeführt werden. In Abbildung sechs rückt die Erinnerungskarriere in den Mittelpunkt. Das Modell ist (im Vergleich zur Medienproduktgeschichte) bewusst auf einem relativ hohen Abstraktionsgrad gehalten. Auffällig ist zunächst, dass die in Abbildung fünf visualisierten Individuuen nicht mehr integriert sind. Außerdem sind die Prozesse nicht detailliert dargestellt, auf eine Partitur wird verzichtet und die lineare Zeitdimension nur mit Fokus auf die fortlaufende Erinnerungskarriere visualisiert. Die Darstellung ist also unterkomplex. Begründet liegt dies in der Komplexität des zu untersuchenden Phänomens und im Zweck des Modells. Durch eine Integration der individuellen Akteure sowie aller Interaktionen würde das Modell die notwendige Übersichtlichkeit verlieren. Individuelle Akteure sind aber bei allen weiteren Schritten mit gedacht. Wechselwirkungen, back loops und verschränkte Zirkelkausalität sind durch die verschiedenen, verschlungenen Kreisläufe integriert, allerdings ohne die sich wandelnden Zustände von Gesamtsystem und Einzelelementen zu visualisieren. Die funktionale Analyse ist auf Theorie-Empirie-Integration angelegt. Ein zu detailliertes Modell würde schnell in den Rang einer Hypothese kommen, was mit dem Zweck der Theoriegenerierung nicht vereinbar wäre. Zudem soll das Modell (nach einer eventuellen Modifikation im weiteren Projektverlauf) dem transkulturellen Vergleich dienen, zu viele Details würden jedoch zu strukturellen Generalisierungen führen. Der primäre Zweck des Modells in diesem Projektstadium ist der einer integrativen Heuristik. 


\section{Abbildung 6: Heuristisches Modell}

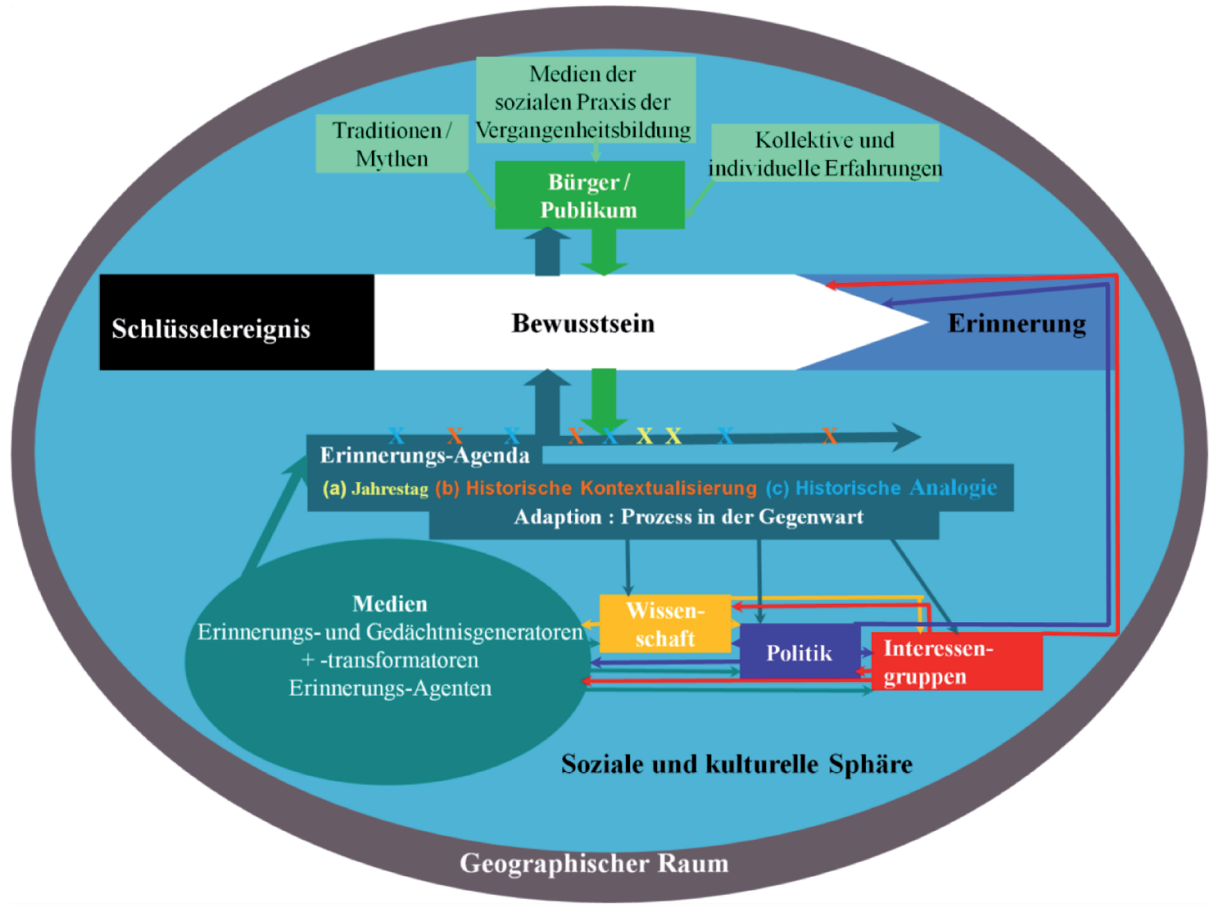

Das heuristische Modell ist die Basis des empirischen Designs der vier Teilstudien und des gesamten StarG-Projekts.

\subsection{Empirische Umsetzung}

Im StarG-Projekt wurden vier integrierte Teilstudien durchgeführt. Integration betrifft einerseits die Einbeziehung der drei Hazarddimensionen Raum, soziokulturelle Sphäre und Zeit und andererseits das zeitliche Nebeneinander der Durchführung der Einzelstudien und ihrer permanenten Abstimmung. Die vier Teilstudien sind:

1) Quantitative Langzeitinhaltsanalyse mit qualitativen Anteilen der erinnernden Berichterstattung in der tagesaktuellen Presse.

2) Dokumentenanalyse des Archivs der Bürgerschaft Hamburg.

3) Experteninterviews mit individuellen Akteuren aus Wissenschaft, Administration und Politik.

Offene Gruppendiskussionen (dokumentarische Methode) mit Bürgern aus Hamburg.

Mit der Inhaltsanalyse wurde die mediale Erinnerung an das Schlüsselereignis seit 1962 rekonstruiert. Es wurde eine quantitative Inhaltsanalyse mit starken qualitativen Anteilen (Möglichkeiten zum offenen Kodieren) durchgeführt. Es 
ging um Diskurse, beteiligte Akteure, Quantität und Qualität der Erinnerung und metaphorische Deutung des Ereignisses. Es wurden drei Tageszeitungen analysiert. Die Raumperspektive wurde bei der Auswahl der Zeitungen berücksichtigt. Es wurden eine regionale Tageszeitung mit Redaktionssitz im Zentrum von Hamburg und Verbreitungsgebiet Metropolregion Hamburg (Hamburger Abendblatt), eine lokale Tageszeitung aus der ländlichen Peripherie von Hamburg (Bergedorfer Zeitung) sowie eine national verbreitete Qualitätszeitung mit weit entferntem Redaktionssitz (Süddeutsche Zeitung) untersucht. In der Zeitdimension stellte der große Zeitraum von 1962 bis zur Datenerhebung 2010 eine Schwierigkeit dar. Es wurden in einem bewussten Auswahlverfahren alle Ausgaben im Zeitraum von zwei Wochen um aus der Vortheorie definierte Erinnerungsereignisse integriert: Jahrestage, folgende schwere Sturmfluten in Hamburg und ausgewählte Flutkatastrophen im Ausland. Die soziokulturelle Dimension wurde mit erinnerungstransformierenden Umständen wie dem aufkommenden Diskurs um den Klimawandel als neue Begründung für Sturmfluten und aktuelle Stadtentwicklungsprojekte wie den Bau der HafenCity in gefährdetes Gebiet integriert. Neben erinnernden Artikeln wurden auch Artikel zu diesen Themen in die Untersuchung integriert. Mit der Dokumentenanalyse wurden politische Entscheidungsprozesse in Hamburg rekonstruiert. Es wurden Dokumente (Plenarprotokolle und Drucksachen) aus dem Archiv der Hamburgischen Bürgerschaft untersucht. Auswahl der untersuchten Dokumente (Entstehungszeit) und Analysemethode (Codebuch) wurden der Pressestudie angepasst. Es ging um Diskussionen und Entscheidungen zum Hochwasserschutz, um die Wahrnehmung der Sturmflutgefahr, um die Verknüpfung von Klima- und Sturmflutdebatte sowie um externe Akteure, die entweder als Experten gehört wurden oder die erwähnt wurden. Besonderes Augenmerk wurde auf eventuelle zeitliche und inhaltliche Parallelen des Medien- und des parlamentarischen Diskurses gelegt. In den Experteninterviews wurden individuelle Akteure aus beteiligten sozialen Feldern befragt. Neben der individuellen Wahrnehmung und Einschätzung der Sturmflutproblematik brachten die Interviews wichtige Kontextinformationen für die Interpretation der anderen Daten. Mit den offenen Gruppendiskussionen wurde schließlich in einer Simulation des Memory Talk die soziale Erinnerung zum Zeitpunkt der Durchführung (Frühjahr 2011) rekonstruiert. Gruppendiskussionen in der dokumentarischen Methode sind bewährt bei der Archäologie von Erinnerung, weil sie durch Selbstläufigkeit in Realgruppen zur Aktivierung von individuellen, verschütteten Erinnerungen führen (Lüthje \& Pater, 2008). Die Gruppendiskussionen waren durch Medienstimuli strukturiert und wurden mit Realgruppen in verschiedenen Hamburger Stadtteilen (Wilhelmsburg, Blankenese und Hafencity) mit unterschiedlichem Gefährdungspotential (Raumdimension) und unterschiedlicher Sozialstruktur (soziokulturelle Dimension) durchgeführt. Die Zeitdimension wurde über die Generationszugehörigkeit der Teilnehmerinnen und Teilnehmer (SeniorInnen und SchülerInnen) integriert.

An dieser Stelle können nicht alle Ergebnisse des Projektes dokumentiert werden. Wichtig ist jedoch, dass die angenommenen Interdependenzen weitestgehend bestätigt wurden. Das Modell musste trotzdem dem Stand der Empirie angepasst werden. Aus kommunikationswissenschaftlicher Sicht sind (zusätzlich zum Journalismus) auch fiktionale Medienerzeugnisse (wie Fernsehfilme, Romane etc.) 
wichtig, deren Veröffentlichung wiederum eine neue Kategorie von Erinnerungsanlass bildet. Die Liste von Erinnerungsanlässen muss also noch erweitert werden. Außerdem muss die Entwicklung des Internet Eingang in das Modell finden. Einerseits wirken auch Internetmedien als Erinnerungsagenten, andererseits findet in sozialen Medien eine besondere Form des Memory Talk statt. Die Funktion des tagesaktuellen Journalismus ist gemäß der Hamburger Studie Sharing, Shaping, Stimulating (3S-Funktion). Erinnerung wird durch die Reaktualiserung des historischen Ereignisses geteilt und transformiert. Gleichzeitig werden Anschlusshandlungen wie erinnernde Gespräche angeregt. Ob diese Funktion auch in anderen Kulturkreisen mit anderen Geohazards gilt, bedarf noch der Untersuchung.

\section{Schluss}

In dem vorliegenden Aufsatz wurde die funktionale Analyse mittlerer Reichweite als Methode einer neuen kulturhistorischen Kommunikationsforschung vorgestellt. Das traditionelle Unvereinbarkeitsdilemma von sozialhistorisch geprägter Kommunikationsgeschichte und neuer Kulturgeschichte sowie der epistemologisch-methodologischen Integration von Individuum und Struktur wurde mit der Theorie komplexer dynamischer Systeme (Mainzer) gelöst. Merkmale komplexer dynamischer Systeme sind: eine Vielzahl von Elementen, Interaktion dieser Elemente im systemischen Zusammenhang, Wandlung und Dynamik, Interdependenz von Gesamtsystem und Einzelelementen, Nichtlinearität, Offenheit der Systeme sowie Austausch mit der Umwelt und Adaption an Umweltbedingungen. Für die Anwendung auf soziokulturelle Systeme ist zu bedenken, dass (1) durch die extreme Verhaltenskomplexität von individuellen Akteuren als relativ eigenständigen Systemelementen quantifizierende Modelle nicht ausreichend sind und (2) komplexe dynamische soziokulturelle Systeme eine eigene epistemologische Fundierung benötigen. Als erkenntnis- und gesellschaftstheoretische Grundlage wurde Bourdieus Strukturkonstruktivismus vorgeschlagen. Auf dieser Basis wurden mit Rückgriff auf Merton und Luhmann der zentrale Funktionsbegriff als intendierte oder nicht intendierte, als latente oder manifeste Folge geklärt und die methodologischen Grundlagen erläutert. Die Anwendbarkeit der Methode wurde an zwei sehr unterschiedlichen Beispielen dargestellt.

Die Programmgeschichte von Klassik Radio war ein Beispiel für Medienproduktgeschichte und damit für die Untersuchung von Medienwandel mit der funktionalen Methode. Es handelt sich um ein originär kommunikationswissenschaftliches, aber intradisziplinär zu realisierendes Konzept, das von einer Einzelperson in einer Qualifikationsarbeit bearbeitet wurde. Es wurde auf strikte TheorieEmpirie-Integration geachtet und dabei mit der Datensammlung begonnen. Das Ergebnis ist ein sehr detailliertes Funktionsmodell. In der Programmgeschichte von Klassik Radio konnten drei Entwicklungsphasen festgestellt werden, deren Vergleich die Identifikation von funktionalen Äquivalenzen ermöglichte.

Das zweite Anwendungsbeispiel für eine funktionale Analyse unterscheidet sich von dem ersten grundsätzlich. Bei der Untersuchung der Entwicklung der sozialen Erinnerung innerhalb eines regionalen Geohazards handelt es sich nicht nur um ein interdisziplinäres, sondern sogar fächergruppenübergreifendes Projekt unter 
Mitwirkung der Kommunikationswissenschaft. Das Projekt wurde von einem interdisziplinär zusammengesetzten Team durchgeführt. Auch hier wurde auf strikte Theorie-Empirie-Integration geachtet, jedoch wurde hier mit Theoriearbeit begonnen. Die Formulierung gemeinsamer Begriffe und die Schaffung einer gemeinsamen Sprache dienen der Integration der beteiligten Disziplinen und Personen in das Projekt. Das Ergebnis der Theoriearbeit war ein vorläufiges Funktionsmodell auf einem relativ hohen Abstraktionsniveau. Auf Visualisierung von Details wurde wegen der großen Komplexität des Forschungsobjekts verzichtet. Dieses Funktionsmodell war als heuristisches Modell Grundlage des empirischen Designs der Teilprojekte im Gesamtprojekt. Das Modell wurde im Projektverlauf dem jeweiligen Erkenntnisstand angepasst, aber trotzdem auf dem hohen Abstraktionsniveau belassen. Das Projekt war explorativ angelegt. Funktionale Äquivalenzen sollen im transkulturellen Vergleich erforscht werden. Ein hoher Abstraktionsgrad verhindert eine Generalisierung von Strukturen ist deshalb von Vorteil für die Adaption des Modells.

Hier soll nicht verschwiegen werden, dass die Durchführung von funktionalen Analysen durchaus problembehaftet sein kann. Im Fall der Realisierung durch eine Einzelperson werden erstens hohe Anforderungen an die methodischen Kompetenzen gestellt. Zweitens sind funktionale Analysen zumeist von hohem Komplexitätsgrad, die Datenmenge ist immens und es ist für Einzelpersonen schwierig, die Übersicht zu behalten. Drittens ist qualitative Modellierung eine interpretative Methode, die (wenn sie nicht durch den Diskurs in einem Team abgesichert wird) einer besonders dichten Datensammlung bedarf, was wieder die Probleme der Methodenkompetenz und der Komplexität betrifft. Durch die Nichtlinearität ist eine exakte Planung erschwert. Diese Risikofaktoren lassen von funktionalen Analysen im Rahmen von Qualifikationsprojekten eher abraten, wenn die Dimension des Forschungsobjekts (zeitlicher Rahmen und Komplexitätsgrad) nicht genau abgeschätzt werden kann. Wichtig ist jedenfalls, dass Doktoranden, die ein solches Projekt bearbeiten, von ihren Betreuern besonders sorgfältig begleitet werden, um bei Problemen frühzeitig eingreifen zu können. Auch interdisziplinäre Projekte können scheitern. Probleme können disziplinäre Egoismen oder unreflektierte habituelle Konflikte sein, die zu permanenten Kämpfen um die Deutungshoheit bzw. symbolischen Kämpfen um die „Leitdisziplin“ führen. Diese Gefahr besteht vor allem dann, wenn die Kommunikation im Projektzusammenhang nicht stetig und eng genug ist, wenn Projekttreffen nur sporadisch und unregelmäßig stattfinden und wenn ausgehandelte Konventionen nicht verschriftlicht werden. Zum Gelingen eines solchen Projektes kann räumliche Nähe aller beteiligten Personen beitragen. Persönliche Voraussetzungen sind Offenheit, extreme wissenschaftliche Neugier und Bereitschaft, sich überraschen zu lassen, Kooperationsbereitschaft, kommunikative Kompetenzen und die Bereitschaft zur Relativierung eigener (sub-)disziplinärer Standards, damit verbunden sind ein hoher Reflexionsgrad, Zuverlässigkeit sowie Anerkennung der Verbindlichkeit von Vereinbarungen und Projektkonventionen.

Wenn jedoch diese Risikofaktoren und Voraussetzungen beachtet werden, bieten funktionale Analysen (sowohl in disziplinären als auch in interdisziplinären Projekten) ein großes Potential, komplexe Zusammenhänge und Prozesse zu er- 
kennen! Auf der disziplinären Ebene gibt die Methode die Möglichkeit der intradisziplinären Zusammenarbeit und der gemeinsamen Theorieentwicklung, was wiederum der Fachentwicklung zugute kommen kann. Auch der persönliche Horizont erfährt Erweiterung. Mit Glück können die in der interdisziplinären $\mathrm{Zu}$ sammenarbeit gewonnenen Anregungen in die eigene Disziplin transferiert werden. So ist z. B. die Autorin erst innerhalb des StarG-Projekts mit der Theorie komplexer dynamischer Systeme vertraut geworden und sie hofft nun, dass diese auch Eingang in den Theoriekanon der Kommunikationswissenschaft findet.

\section{Literatur}

Arnold, K., Behmer, M., \& Semrad, B. (Hrsg.). (2008). Kommunikationsgeschichte. Positionen und Werkzenge. Ein diskursives Hand- und Lehrbuch. Berlin: Lit-Verlag.

Bar-Yam, Y. (1997). Dynamics of Complex Systems. Reading, Massachesetts: AddisonWesley.

Beck, U. (2007). Weltrisikogesellschaft. Frankfurt/Main: Suhrkamp.

Behmer, C. (2008). Quellen selbst erstellen. Grundzüge, Anwendungsfelder und Probleme von Oral History in der medien- und kommunikationsgeschichtlichen Forschung. In K. Arnold, M. Behmer, \& B. Semrad (Hrsg.), Kommunikationsgeschichte. Positionen und Werkzenge. Ein diskursives Hand- und Lehrbuch (S. 343-362). Berlin: Lit-Verlag.

Bösch, F., \& Danyel, J. (Hrsg.) (2012). Zeitgeschichte - Konzepte und Methoden. Göttingen: Vandenhoeck \& Ruprecht.

Bourdieu, P. (1974). Zur Soziologie der symbolischen Formen. Frankfurt/Main: Suhrkamp. (Original: 1970).

Bourdieu, P. (1976). Entwurf einer Theorie der Praxis auf der ethnologischen Grundlage der kabylischen Gesellschaft. Frankfurt/Main: Suhrkamp. (Original: 1972).

Bourdieu, P. (1985). Sozialer Raum und „Klassen“. Leçon sur la leçon. Zwei Vorlesungen. Frankfurt/Main: Suhrkamp. (Original: 1984 und 1982).

Bourdieu, P. (1987). Die feinen Unterschiede. Kritik der gesellschaftlichen Urteilskraft. Frankfurt/Main: Suhrkamp. (Original: 1979).

Bourdieu, P. (1992a). Rede und Antwort. Frankfurt/Main: Suhrkamp. (Original: 1987).

Bourdieu, P. (1992b). Sozialer Raum und symbolische Macht. In ders., Rede und Antwort (S. 135-154). Frankfurt/Main: Suhrkamp.

Bourdieu, P. (1992c) . Das Interesse des Soziologen. In P. Bourdieu, Rede und Antwort (S. 111-118). Frankfurt/Main: Suhrkamp.

Bourdieu, P. (1992d). Meinungsforschung - Eine Wissenschaft ohne Wissenschaftler. In P. Bourdieu, Rede und Antwort (S. 208-218). Frankfurt/Main: Suhrkamp.

Bourdieu, P. (1993). Sozialer Sinn. Kritik der theoretischen Vernunft. Frankfurt/Main: Suhrkamp. (Original: 1980).

Bourdieu, P. (1998). Der doppelte Bruch. In P. Bourdieu, Praktische Vernunft. Zur Theorie des Handelns (S. 83-95). Frankfurt/Main: Suhrkamp.

Brandau, M. (2010). Modellierung und Netzwerkanalyse komplexer Systeme sozialen und polymeren Ursprungs. Dissertation Martin-Luther-Universität Halle-Wittenberg.

Burke, P. (2005). Was ist Kulturgeschichte? Frankfurt/Main: Suhrkamp.

Classen, C. (2008). Qualitative Diskursanalysen in der historischen Medien- und Kommunikationsforschung. In K. Arnold, M. Behmer, \& B. Semrad (Hrsg.), Kommunikations- 
geschichte. Positionen und Werkzeuge. Ein diskursives Hand-und Lehrbuch (S. 363382). Berlin: Lit-Verlag.

Confino, A. (1997). Collective Memory and Cultural History: Problems of Method. American Historical Review, 102, 1386-1403.

Coser, L. A. (2000). Robert K. Merton. In D. Kaesler (Hrsg.), Klassiker der Soziologie, Band 2: Von Talcott Parsons bis Pierre Bourdieu (S. 152-170). München: C. H. Beck.

Daniel, U. (2001). Kompendium Kulturgeschichte. Theorien, Praxis, Schlüsselwörter. Frankfurt/Main: Suhrkamp.

Daniel, U. (2003). Kulturgeschichte. In A. Nünning, \& V. Nünning (Hrsg.), Konzepte der Kulturwissenschaften (S. 186-204). Stuttgart: Metzler.

Drews, W., \& Oesterle, J. R. (Hrsg.). (2008). Transkulturelle Komparatistik. comparativ, $18(3-4)$.

Elias, N. (1997). Über den Prozess der Zivilisation. Soziogenetische und psychogenetische Untersuchungen. Erster Band: Wandlungen des Verhaltens in den weltlichen Oberschichten des Abendlands. Frankfurt/Main: Suhrkamp. (Original: 1939).

Faulstich, W. (1991). Einfùhrung. In W. Faulstich. (Hrsg.), Medien und Kultur (S. 7-15). Göttingen: Vandenhoeck \& Ruprecht.

Faulstich, W. (1996). Medien und Öffentlichkeiten im Mittelalter: 800 - 1400. Göttingen: Vandenhoeck \& Ruprecht.

Faulstich, W. (1997). Das Medium als Kultur: von den Anfängen bis zur Spätantike (8. Jahrhundert). Göttingen: Vandenhoeck \& Ruprecht.

Faulstich, W. (1998). Medien zwischen Herrschaft und Revolte: die Medienkultur der frühen Neuzeit (1400 - 1700). Göttingen: Vandenhoeck \& Ruprecht.

Faulstich, W. (2002). Die bürgerliche Mediengesellschaft (1700-1830). Göttingen: Vandenhoeck \& Ruprecht.

Faulstich, W. (2004). Medienwandel im Industrie- und Massenzeitalter (1830 - 1900). Göttingen: Vandenhoeck \& Ruprecht.

Fröhlich, G. (1994). Kapital, Habitus, Feld, Symbol. Grundbegriffe der Kulturtheorie bei Pierre Bourdieu. In I. Mörth, \& G. Fröhlich (Hrsg.), Das symbolische Kapital der Lebensstile (S. 31-54). Frankfurt/Main: Campus.

Früh, W., \& Schönbach, K. (1982). Der dynamisch-transaktionale Ansatz. Ein neues Paradigma der Medienwirkungen. Publizistik, 27(1-2), S. 74-88.

Geertz, C. (1983). Dichte Beschreibung. Frankfurt/Main: Suhrkamp.

Gries, R. (2008). Kulturgeschichte des Kommunizierens. Konjunktionen, Konjunkturen und Konnektivitäten. In K. Arnold, M. Behmer, \& B. Semrad (Hrsg.), Kommunikationsgeschichte. Positionen und Werkzeuge. Ein diskursives Hand- und Lehrbuch (S. 45-72). Berlin: Lit-Verlag.

Hartmann, H. (Hrsg.). (1967). Moderne Amerikanische Soziologie. Neuere Beiträge zur soziologischen Theorie. Stuttgart: Enke.

Hunt, L. (1998). Psychologie, Ethnologie und „linguistic turn“ in der Geschichtswissenschaft. In H.-J. Goertz (Hrsg.), Geschichte. Ein Grundkurs (S. 671-693). Rowohlt: Reinbek bei Hamburg.

Hunt, L. (Hrsg.). (1989). The New Cultural History. Berkeley: University of California Press. 
Jaeger, F. (2004). Historische Kulturwissenschaft. In F. Jäger, \& J. Straub (Hrsg.), Handbuch der Kulturwissenschaften, Band 2 Paradigmen und Disziplinen (S. 518-545). Stuttgart: Metzeler.

Jordan, S. (2010). Theorien und Methoden der Geschichtswissenschaft. Paderborn: Schöningh.

Karmasin, M. \& Winter, C. (Hrsg). (2003). Kulturwissenschaft als Kommunikationswissenschaft. Projekte, Probleme und Perspektiven. Wiesbaden: Westdeutscher Verlag.

Kinnebrock, S. (2008). Gender matters! Oder inwieweit die Kommunikationsgeschichte von der Frauen- und Geschlechtergeschichte profitieren kann. In K. Arnold, M. Behmer, $\&$ B. Semrad (Hrsg.), Kommunikationsgeschichte. Positionen und Werkzenge. Ein diskursives Hand- und Lehrbuch (S. 209-234). Berlin: Lit-Verlag.

Koch. H. J., \& Glaser, H. (2005). Ganz Ohr. Eine Kulturgeschichte des Radios in Deutschland. Köln u.a.: Böhlau.

Lamprecht, K. (1912). Einführung in das historische Denken. Leipzig: Voigtländer.

Lazarsfeld, P. F., \& Merton, R. K. (1948). Mass communication, popular taste, and organized social action. In L. Bryson (Hrsg.), The communication of ideas (S. 95-118). New York: Harper.

Lewin, R. (1993). Die Komplexitätstheorie. Wissenschaft nach der Chaosforschung. Hamburg: Hoffmann und Campe.

Löblich, M. (2010). Die empirisch-sozialwissenschaftliche Wende. Ein Beitrag zur historischen und kognitiven Identität der Kommunikationswissenschaft. Medien \& Kommunikationswissenschaft, 58(4), 544-562.

Luhmann, N. (1987). Soziale Systeme. Grundriß einer allgemeinen Theorie. Frankfurt/ Main: Suhrkamp.

Luhmann, N. (2003). Soziologie des Risikos. Berlin: de Gruyter.

Luhmann, N. (2005). Funktion und Kausalität. In N. Luhmann, Soziologische Aufklärung 1 (S. 11-38). Frankfurt/Main: Suhrkamp. (Original: 1962).

Lüthje, C. (2008). Das Medium als symbolische Macht. Untersuchung zur soziokulturellen Wirkung von Medien am Beispiel von Klassik Radio. Dissertation Universität Hamburg. BoD: Norderstedt.

Lüthje, C. (2010a). Kulturradio in Deutschland 2010: Versuch einer Bestandsaufnahme. Publizistik, 55(1), 23-39.

Lüthje, C. (2010b). Öffnung von Elitekultur durch massenmedial verbreitete symbolische Innovation? Klassische Musik im privat-kommerziellen Radio in Deutschland. Medien \& Kommunikationswissenschaft, 58(1), 46-62.

Lüthje, C., \& Pater, M. (2008). Das Gruppendiskussionsverfahren in der dokumentarischen Methode. Chancen für die historische Rezeptionsforschung am Beispiel der Domestizierung des Radios in den 1950er Jahren. Rundfunk und Geschichte, 34(3-4), 5-15.

Mainzer, K. (2004). Was sind komplexe Systeme? Komplexitätsforschung als integrative Wissenschaft. Abgerufen von http://www.integrative-wissenschaft.de/Archiv/dokumente/Mainzer-14_10_04.pdf [19.02.2013].

Mainzer, K. (2008). Komplexität. Paderborn: Fink.

Merten, K. (2007). Einführung in die Kommunikationswissenschaft. 3. Auflage. Berlin: LIT-Verlag.

Merton, R. K. (1967). Funktionale Analyse. In H. Hartmann (Hrsg.), Moderne Amerikanische Soziologie. Neuere Beiträge zur soziologischen Theorie (S. 119-150). Stuttgart: Enke. 
Merton, R. K. (1995). Soziologische Theorie und soziale Struktur. Berlin: de Gruyter.

Neverla, I. (2009). Media Coverage on regional Geohazards - and its role in the collective memory. Paper presented to IAMCR-Conference, Mexico-City.

Münch, R. \& Schmidt, J. (2005). Medien und sozialer Wandel. In M. Jäckel (Hrsg.), Lehrbuch der Mediensoziologie (S. 201-218). Wiesbaden: VS-Verlag.

Nünning, V. (2005): New Cultural History/Kulturgeschichte. In A. Nünning (Hrsg.), Grundbegriffe der Kulturtheorie und Kulturwissenschaften (S. 165-168). Stuttgart: Metzeler.

Papilloud, C. (2003). Bourdieu lesen. Einführung in eine Soziologie des Unterschieds. Bielefeld: Transcript.

Peirce, C. S. (1983). Phänomen und Logik der Zeichen. Frankfurt/Main: Suhrkamp. (Original: 1903).

Peltonen, M. (2004). From Discourse to Dispositif: Michel Foucault's Two Histories. Historical Reflections Reflexions Historiques, 30(2), 205-219.

Petak, W. J. \& Atkisson, A. A. (1982). Natural Hazard Risk Assesment and Public Policy. Anticipating the Unexpected. Springer: New York.

Pfister, C. (2009). The "disaster gap" of the 20th century and the loss of traditional disaster memory. GAIA 18(3), 239-246.

Raphael, L. (2003). Historische Anthropologie und neue Kulturgeschichte. In ders., Geschichtswissenschaft im Zeitalter der Extreme. Theorien, Methoden, Tendenzen von 1900 bis zur Gegenwart (S. 228-246). München: Beck.

Ratter, B. (2009). People's perception of natural hazards. Paper presented at the 6 th International SedNet conference, Hamburg.

Rehbein, B. (2006). Die Soziologie Pierre Bourdieus. Konstanz: UVK.

Reinhardt, J. D., \& Jäckel, M. (2005). Massenmedien als Gedächtnis- und Erinnerungs,generatoren'. Mythos und Realität einer ,Mediengesellschaft'. In: P. Rössler, \& F. Krotz (Hrsg.), Mythen der Mediengesellschaft (S. 93-112). Konstanz: UVK.

Robinson, S. (2009). „We all were there“; Remembering America in the anniversary coverage of Hurricane Katrina. Memory Studies, 2, 235-253.

Rühl, M. (2008). Kommunikationskulturen der Weltgesellschaft. Theorie der Kommunikationswissenschaft. Wiesbaden: VS Verlag.

Rühl, M. (2011). Journalistik und Journalismen im Wandel. Eine kommunikationswissenschaftliche Perspektive. Wiesbaden: VS-Verlag.

Saxer, U. (1998). Zur Theorie von Medien-Kulturkommunikation. In: U. Saxer (Hrsg.), Medien-Kulturkommunikation (S. 9-43). Wiesbaden: Westdeutscher Verlag.

Saxer, U. (2012). Mediengesellschaft. Eine kommunikationssoziologische Perspektive. Wiesbaden: VS-Verlag für Sozialwissenschaften.

Simonson, P. (2005). The serendipity of Merton's communications research. International Journal of Public Opinion Research, 17(1). Doi: 10.1093/ijpor/edho68

Schmidt, S. J. (1991). Medien, Kultur: Medienkultur. In: Faulstich, W. (Hrsg.), Medien und Kultur (S. 30-50). Göttingen: Vandenhoeck \& Ruprecht.

Schmidt, S. J. (2003). Kognitive Autonomie und soziale Orientierung. Konstruktivistische Bemerkungen zum Zusammenhang von Kognition, Kommunikation, Medien und Kultur. Münster: LIT-Verlag.

Schwingel, M. (2005). Pierre Bourdieu zur Einführung. 5. Auflage. Hamburg: Junius. 
Sturm, R., \& Zirbik, J. (1996). Die Radio-Station. Ein Leitfaden für den privaten Hörfunk. Konstanz: UVK.

Ulbig, E., Hertel, R. F., \& G.-F. Böl (Hrsg.). (2009). Evaluierung der Kommunikation über die Unterschiede zwischen "risk" und „hazard“. Abschlussbericht. BfR Wissenschaft H. 2.

Wacquant, L. (2003). Eine Grammatik der Praxis im Handeln. In C. Papilloud, Bourdieu lesen. Einführung in eine Soziologie des Unterschieds (S. 107-111). Bielefeld: Transcript.

Welzer, H. (2001). Das soziale Gedächtnis. In H. Welzer (Hrsg.), Das soziale Gedächtnis. Geschichte, Erinnerung, Tradierung (S. 9-21). Hamburg: Hamburger Edition.

Winter, C. (2003). Der Zusammenhang von Medienentwicklung und Wandel als theoretische Herausforderung. Perspektiven für eine artikulationstheoretische Ergänzung system-funktionaler Analysen. In M. Behmer (Hrsg.), Medienentwicklung und gesellschaftlicher Wandel. Beiträge zu einer theoretischen und empirischen Herausforderung (S. 65-101). Wiesbaden: Westdeutscher Verlag.

Winter, C. (2006). Die Medienkulturgeschichte des christlichen Predigers von den Anfüngen bis heute. Graz: Nausner \& Nausner.

Winter, C. (2012). Funktionale (Medienkultur-)Gesellschaftsgeschichte und die Entwicklung von Medien In M. Karmasin \& C. Winter (Hrsg.), Analyse, Theorie und Geschichte der Medien. Festschrift für Werner Faulstich (S. 195-213). München: Wilhelm Fink.

Zelizer, B. (2008). Why memory's work on journalism does not reflect journalism's work on memory. Memory Studies, 1, 79-87.

Zierold, M. (2006). Gesellschaftliche Erinnerung. Eine medienkulturwissenschaftliche Perspektive. Berlin: de Gruyter.

Zwicky, F. (1969). Discovery, Invention, Research - Through the Morphological Approach. Toronto: Macmillian. 


\section{EXTENDED ABSTRACT}

\section{Functional Analysis as Method of New Cultural Historical Communication Research. Methodology, Epistemology and Sample Applications}

\section{Corinna Lüthje}

The goals of this paper are: (1) To present functional analysis as a suitable method for the integration of new cultural history and the history of communication in empirical research and for the generation of theories, $(2)$ to explain the operationalization, and (3) to illustrate the merits of this method. In order to do so, however, epistemological issues must first be addressed:

(a) The issue of the theoretical integration of the actor perspective and systemic structure.

(b) The issue of the integration of theory and empiricism.

(c) The issue of structural determinism.

(d) The dimensions of the question at hand.

To address these issues, the epistemological and methodological combination of three components is proposed in this paper:

(1) The research program for functional analyses of the middle range according to Robert K. Merton with methodological additions from an early work by Niklas Luhmann.

(2) The theory of complex dynamic systems or complexity theory for short (here, the version by Klaus Mainzer was used).

(3) Structural constructivism as the epistemological quintessence of Pierre Bordieu's reflexive social theory.

Merton argued against structural functionalism. He also departed from an allencompassing grand theory. He strove to develop middle range theories. Merton distinguished the term function as a consequence from the intended targetedness in meaning as role. Furthermore, he also recognized that functions can be assumed by multiple elements for the preservation of a superordinate coherence of elements (i.e. that of a system). A single element can have multiple functions. Hence, he introduced the term functional equivalence. Furthermore, he recognized that, in addition to positive functions, there are also dysfunctions that must also be accounted for during analysis. He distinguished between manifest and latent functions. According to Merton's concept, sociological theory construction and empirical research should be closely linked. Merton turned the functional analysis into a flexible instrument for the generation of theories based on empirical data. The introduction of reciprocity and hence the relativization of the principle of causality was Luhmann's crucial addition. 
The terminological unity and the rigidity of grand sociological theories are in opposition to openness and adaptability. The theory of complex dynamic systems is characterized by an extremely low degree of elaboration. It is reduced to the naming of principal elements and processes. This makes it a flexible instrument that can be applied to a wide spectrum of phenomena in almost all disciplines and interdisciplinary contexts - just like functional analysis. At the same time, with this concept that has been reduced to the bare essentials, it is possible to explain highly complex relationships. The characteristics of complex dynamic systems are: a multitude of elements, the interaction of these elements in a systemic context, transformation and dynamism, interdependence between the overall system and single elements, non-linearity, openness of the systems as well as exchanges with the environment and adaptation to environmental conditions. However, for each form of system, a special set of terms must be found, or the definition of terms must be adapted. For application to socio-cultural systems, it should be noted that (1) due to the extremely complex behaviour of individual actors as relatively independent system elements, quantificational models are insufficient, and that (2) complex and dynamic socio-cultural systems require their own epistemological foundation. To address this, Pierre Bourdieu's structural constructivism will be proposed in which actors and social structures are seen as being independent of each other. This will address the problems of structural determinism and the integration of the individual and structure. In addition, Bourdieu abstained from describing a grand theory in full, instead determining the interdependent individual elements of his concept of culture and society as flexible instruments, as well as promoting and practicing strict theoretical empiricism.

This methodological text is split into two parts: (a) The presentation of three epistemological and methodological theory elements which are relevant for the application of the functional method in the context of new cultural historical communication research followed by (b) a presentation of two application samples. These usage examples are meant to serve as loose guidelines for carrying out functional analyses. With their help, the (possible) sequence of steps for studies is demonstrated. This is not an instructional manual with strict requirements that to be understood literally, as each new project has its own requirements which have to be taken into account when selecting a method for collecting data and defining terms. Because the three theory elements are each to be understood as flexible instruments that can be applied to a wide array of questions and issues, it is mainly original source texts that are excerpted in the theoretical section. The aim is to achieve a concise representation that remains unaltered as far as possible. The integration of elements takes place during use. With the two usage examples of media product history and reconstruction of the creation of social memory from the author's research activities, the merits of functional analysis will be demonstrated for new cultural historical communication research.

The program history of Klassik Radio is an example of history of media products and hence also an example for the investigation of media through the ages with the functional method. This is an original communication studies project that is to be realized in an interdisciplinary manner, which an individual worked on for the purposes of a qualifying dissertation. Great attention was paid to the 
strict integration of theory and empiricism during data collection. The result is an extremely detailed functional model. In the Klassik Radio program history, three developmental phases could be identified, and the comparison of these phases allowed for the identification of functional equivalences.

The second application sample for a functional analysis is fundamentally different from the first. In this case, the function of media-based communication during the creation of social memory in the context of hazard research will be investigated. The concept of a regional geohazard is not only interdisciplinary, but also inherently combines multiple subject groups through the integration of the natural sciences, sociology and cultural studies. Here, communication is the key principle. This project is concerned with the contribution of media communication to societal change. In this case, the functional analysis is also a helping hand for ensuring the success of interdisciplinary cooperation. The project was carried out by an interdisciplinary team. Here too, great attention was paid to a strict integration of theory and empiricism, but at this juncture the theoretical work began. The formulation of common terms and the creation of a common language served to integrate the disciplines involved and the persons into the project. The result of the theoretical work was a preliminary functional model at a rather high level of abstraction. Due to the high complexity of the research objective, a visualization of details was not performed. As a heuristic model, this functional model formed the basis of the empirical design of the sub-projects in the overall project. During the course of the project, the model was adapted to the respective level of knowledge. Despite this, it was left at a high level of abstraction. The project was created to be exploratory. Functional equivalences are to be investigated in a transcultural comparison. A high level of abstraction prevents a generalization of structures, which makes it advantageous for the adaption of the model.

This paper concludes with a critical evaluation of the performance and practicability of the method. 